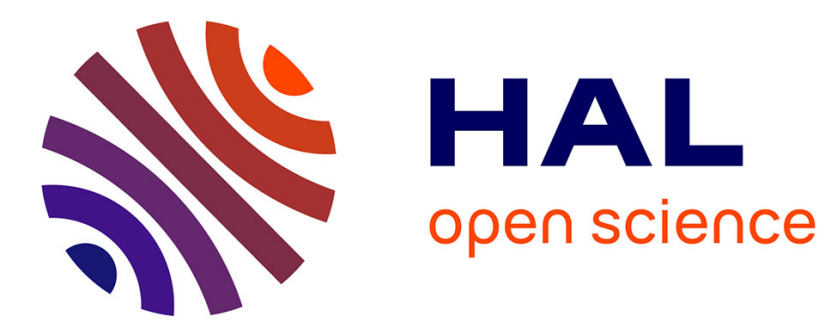

\title{
Homology modeling of 2C-methyl-erythritol-4-phosphate cytidylyltransferase, the third enzyme in the MEP pathway for isoprenoid biosynthesis
}

Cristian Obiol-Pardo, Alex Cordero, Jaime Rubio-Martinez, Santiago Imperial

\section{To cite this version:}

Cristian Obiol-Pardo, Alex Cordero, Jaime Rubio-Martinez, Santiago Imperial. Homology modeling of 2C-methyl-erythritol-4-phosphate cytidylyltransferase, the third enzyme in the MEP pathway for isoprenoid biosynthesis. Journal of Molecular Modeling, 2009, 16 (6), pp.1061-1073. 10.1007/s00894009-0615-x . hal-00568334

\section{HAL Id: hal-00568334 \\ https://hal.science/hal-00568334}

Submitted on 23 Feb 2011

HAL is a multi-disciplinary open access archive for the deposit and dissemination of scientific research documents, whether they are published or not. The documents may come from teaching and research institutions in France or abroad, or from public or private research centers.
L'archive ouverte pluridisciplinaire HAL, est destinée au dépôt et à la diffusion de documents scientifiques de niveau recherche, publiés ou non, émanant des établissements d'enseignement et de recherche français ou étrangers, des laboratoires publics ou privés. 


\section{Editorial Manager(tm) for Journal of Molecular Modeling}

Manuscript Draft

Manuscript Number: JMM0920R1

Title: Homology modeling of Mycobacterium tuberculosis 2C-methyl-D-erythritol-4-phosphate cytidylyltransferase, the third enzyme of the MEP pathway for isoprenoid biosynthesis

Article Type: Original paper

Keywords: 2C-methyl-D-erythritol-4-phosphate cytidylyltransferase (CMS); homology modeling; molecular dynamics; drug design; tuberculosis

Corresponding Author: Dr. Santiago Imperial, Ph.D.

Corresponding Author's Institution: University of Barcelona

First Author: Cristian Obiol-Pardo

Order of Authors: Cristian Obiol-Pardo; Alex Cordero; Jaime Rubio-Martinez, PhD; Santiago Imperial, Ph.D.

Abstract: Tuberculosis is one of the leading infection diseases in humans. Discovering new treatments for this disease is urgent, especially due to the emergence of multiple drug resistant organisms and to reduce the total duration of the current treatments. In Mycobacterium tuberculosis, the synthesis of isoprenoids has been reported as an interesting pathway to target and especial attention has been focused on the methylerythritol phosphate (MEP) pathway comprising the early steps of biosynthesis. In this context, we have studied the enzyme 2C-methyl-D-erythritol-4-phosphate cytidylyltransferase (CMS), the third enzyme of the MEP pathway for the biosynthesis of isoprenoid precursors, since so far there is no resolved structure of this protein in M. tuberculosis, and its use as a drug target is therefore seriously limited. We have performed a homology modeling of the M. tuberculosis CMS to provide a reliable model to be used in structure-based drug design. After evaluating the quality of the model, we fully studied the catalytic site and the dimerization interface of the model, suggesting the most important sites (conserved and non-conserved) useful for drug discovery and mutagenesis studies. We found that the metal coordination of CDP-methylerythritol is substantially different in the M. tuberculosis CMS with respect to the E. coli variant, corroborating that the former is able to utilize several metal ions for catalysis. Moreover, we propose that electrostatic interactions could explain the higher affinity of the MEP substrate compared with the CTP substrate in the M. tuberculosis enzyme as reported previously.

Response to Reviewers: Reviewer \#1: This paper presents molecular modelling work on Mycobacterium tuberculosis 2C-methyl-D-erythritol-4-phosphate cytidylyltransferase (CMS) enzyme. Homology model was constructed following by detailed investigation of the catalytic site and dimerization interface of the model.

So far, no homology model of M. tuberculosis CMS has been published.

The major flaw of the homology modelling part is that in contrast to the authors' statement, there are more than 3 available templates (see 2VSH, 2VSI,3F1C, 1W55,1W57). Moreover, while the authors state that the template was chosen based on the lowest resolution, the template used for E. coli is not the one with the lowest resolution (see 1I52).The fact that more templates could be used raises concerns regarding the authors' knowledge in this field. Additonally, because of the availability of more 
templates than the authors mentioned, the usefullness of homology modelling on multiple templates cannot be ruled out.

We were aware of most of the structures that the Referee noticed, but for presenting functional differences or for not having the adequate cofactor, these structures were not considered in the first version of the manuscript.

With respect to $1 \mathrm{~W} 55$ and 1W57, both structures correspond to the bifunctional IspDF enzyme. This enzyme contains two catalytical domains, one is effectively involved in the third reaction of the MEP pathway, but the second one catalyzes stage five, that yields a cyclic product (cMEDP) instead of CDP-ME. We considered that the presence of this second domain might affect the use and the value of these structures as templates to model M. tuberculosis CMS.

Codes 2VSH, 2VSI and 3F1C are effectively transferases but in Gram-positive organisms and correspond to the IspD type2 (our model is the type 1 ). They were crystallized in absence of the cofactor (3F1C) or with cofactors that are not involved in the MEP pathway (CDP-ribitol for 2VSH, and $\mathrm{CDF}$ for $2 \mathrm{VSI}$ ). In fact $2 \mathrm{VSH}$ and $2 \mathrm{VSI}$ correspond to a protein which was isolated and characterized as an enzyme involved in the biosynthesis of teichoic acid which later on was shown to catalyze also the IspD reaction in the MEP pathway. Therefore, these structures were not considered at the time our study was started as good templates for M. tuberculosis CMS, specially to study it in complex with CDPME.

Finally, it is true that E. coli $1 \mathrm{I} 53$ has a better resolution, but this CMS does not contain the CDP-ME cofactor, but only one of the reactive substrates, the CTP. We were also interested in the interactions coming from the MEP fragment and therefore our E. coli template (1INI), that contains effectively both fragments (as the CDP-ME), was optimum for our study of the whole catalytical site.

After pondering these available structures, we still consider that the selected template from E. coli is the best one for obtaining the M. tuberculosis CMS model reported and for studying its catalytical site. This point was examined carefully being very important for the homology modeling. Sequence identity also favours the election of the E. coli template. For instance the critical residues P13, A14, A15, G16, G18, K27, A107, R109, T140, T165 and K213 are all conserved between the E. coli and the M. tuberculosis enzymes. This is not the case for the Thermus thermophilus CMS, for which A107 is not conserved, or for the Thermotoga maritima CMS, for which P13 and A107 are not conserved. Additionally, in the structures that the Referee noticed, sequence identity is slightly inferior:

Identity of M. tuberculosis with :

E. coli Template (1INI, 1I53) $=32 \%$

Campylobacter jejuni $(1 \mathrm{~W} 55,1 \mathrm{~W} 57)=26.4 \%$

Listeria monocytogenes $(3 \mathrm{~F} 1 \mathrm{C})=26.2 \%$

Streptococcus pneumoniae $(2 \mathrm{VSH}, 2 \mathrm{VSI})=24.6 \%$

Following the comments of the Referee we have expanded the Methods, section 2.1 by superimposing all these possible structures to the one used as template (1INI), thus we have modified the text and Fig. 2A. Similarly to our first version considering the E. coli, Thermus thermophilus, and Thermotoga maritima, the other structures are also highly similar (presenting RMS between 4.5 to $6.0 \AA$ ). As we already stated in the first version, the election of other templates of this group should produce a very similar M. tuberculosis CMS model. Also note that the MD simulation overcomes these RMS deviations. All in all, as we needed a structure in complex with the CDP-ME and taking into account the above comments, we still consider that the selected template (and its further study) is the most adequate to 
build the M. tuberculosis CMS model, as part of the MEP pathway. We are grateful to the Referee because the new version is more complete in discussing possible templates.

The other major flaw of the paper is the evaluation of the binding energy of the ligands MEP and CPT. Although the authors state that the experimentally determined binding affinity of the substrates are 43 and $92 \mathrm{uM}$, respectively. In the paper the authors calculate and discuss a binding energy as low as about $-110 \mathrm{kcal} / \mathrm{mol}$ (Figure 11). Considering that CC bond energy is about $-80 \mathrm{kcal} / \mathrm{mol}$, this value is highly unrealistic and shows absolutely no correlation with the experimentally determined value (which would be about $-6 \mathrm{kcal} / \mathrm{mol}$ in DG).

It is true that the calculated values of the binding free energy using the MMGBSA protocol are unrealistic for the cofactor bound to CMS. The method was originally developed to calculate precise values of the experimental free energies, within an error of $1-2 \mathrm{kcal} / \mathrm{mol}$ (see an early work in J Comput Chem 25, 238-250, 2004). However, when more systems were treated by using this method, authors noticed that the real useful application of MMGBSA (or MMPBSA) is to account for relative binding free energies contributions to binding. For instance in a recent paper in J Chem Inf Model 49, 1033-1048, 2009 the authors stated that 'to reproduce the absolute values of binding free energies may not be the primary aim for applying the MMGBSA protocol'.

Moreover, it is usual to find overestimated values of the calculated binding free energy: for instance $99.23 \mathrm{kcal} / \mathrm{mol}$ in J Chem Inf Model 48, 2030-2041, 2008 or $-58.56 \mathrm{kcal} / \mathrm{mol}$ in J Med Chem 48, 77967807,2005 . We previously studied a highly charged cofactor (thiamine pyrophosphate) in complex with a protein, quoted as ref. 51 in the paper, finding similarly high numerical values (between -78.72 and $-155.64 \mathrm{kcal} / \mathrm{mol}$ ). In ref. 51 we suggested that these large values are a consequence of the electrostatic energy extracted from the force field that is not well counterbalanced by the desolvation penalty using the GB (or PB) approach and different sets of radii.

Unfortunately, this limitation has not been corrected so far in this methodology, although MMGBSA has been proven very useful to correlate relative binding free energies and to identify the main nature of binding (electrostatic, hydrophobic, entropically driven...).

Since the MMGBSA method overestimates the experimental energy, we used a bar graphics for the contributions of the cofactor binding instead of using a numerical table and we tried not to mention the precise values. Nevertheless, as your appreciation is adequate and the high energies are confusing, the new version of the manuscripts includes a brief discussion (Methods, section 2.3 and Results, section 3.2) about the limitations of the method, specially remarking that the absolute values are not well reproduced.

Minor points

There are too many figures in the paper, some of them could be omitted. As an example, Figure 3, 4 and 6 could be discussed in the text only, figure presentation does not add much value to the understanding.

We have removed Figures 3 and 4 in the revised version. We prefer not to remove Figure 6 because it is more clear to show an image of the Prosa profile changes before and after MD, than trying to remark this subtle changes in the text.

Moreover, it is quiet confusing that the residue numbering does not restart in case of the dimers (e.g. Figure 5). As a result, some figures indicate 220 amino acid residues, whereas some indicate 450.

This error has been corrected in the new version.

What does the gray color mean in Figure 7 ? 
The grey colour was the cartoon representation of the helix formed after MD, but shown in transparent. The revised version includes a more clear representation of the helix formed after MD.

It is hard to see the contribution of individual amino acids in Figure 9- a table would be more informative.

Accordingly to the Referee's comment, we have removed Fig. 9 and added the information into a new table, Table 1. 


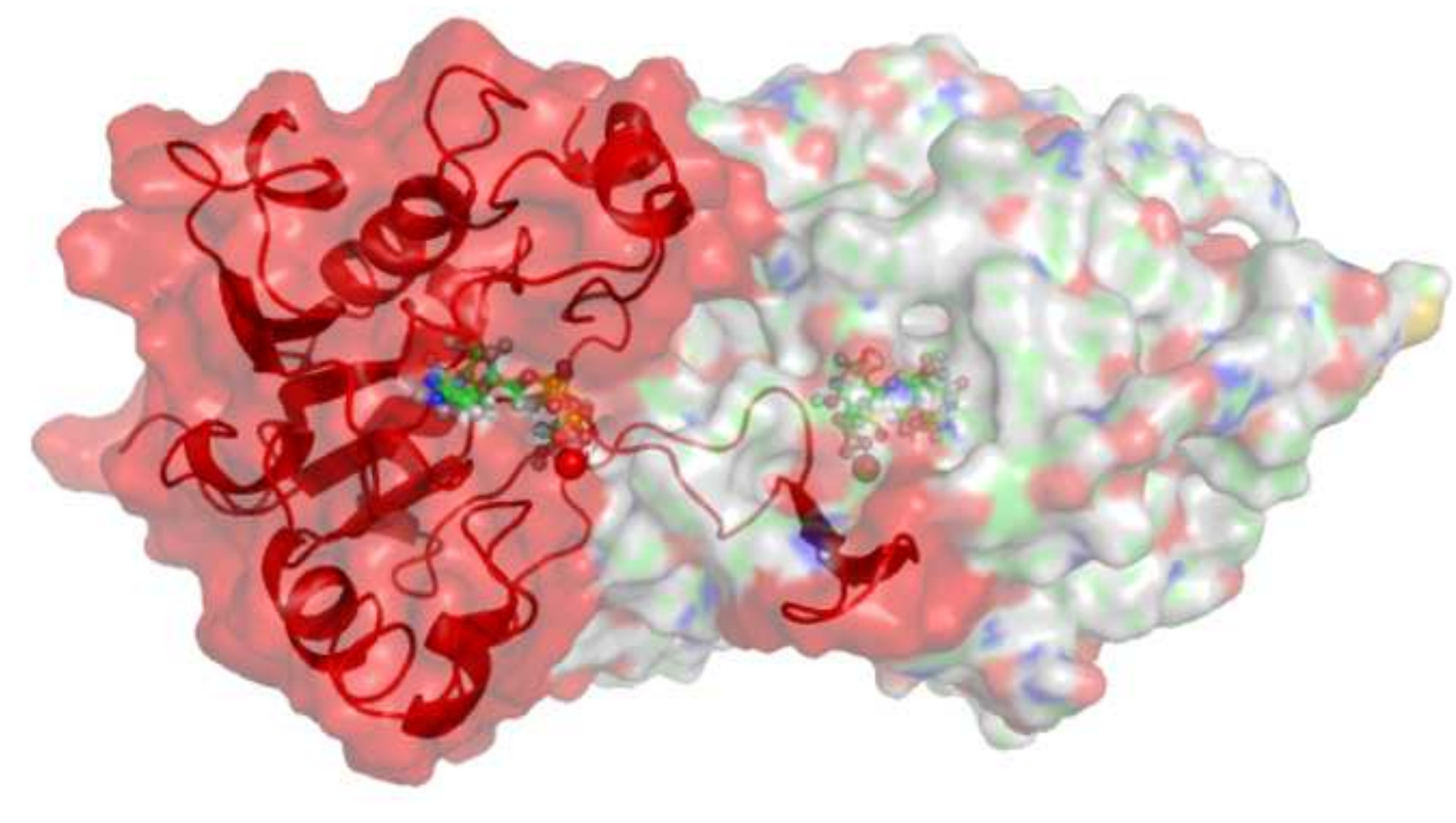

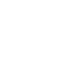$$
\text { ofecio }
$$
. 


\title{
Homology modeling of $M$. tuberculosis 2C-methyl-D-erythritol-4- phosphate cytidylyltransferase, the third enzyme of the MEP pathway for isoprenoid biosynthesis
}

Received: 15.07.2009 / Accepted: 20.10.2009

Cristian Obiol-Pardo ${ }^{1}$, Alex Cordero $^{2}$, Jaime Rubio-Martinez $^{1}$, Santiago Imperial $^{2, 凶}$

${ }^{1}$ Dept. de Química Física, Universitat de Barcelona (UB) and the Intitut de Recerca en Química Teòrica i Computacional (IQTCUB). Martí i Franquès 1, 08028, Barcelona, Spain

${ }^{2}$ Dept. de Bioquímica i Biologia Molecular, Universitat de Barcelona (UB). Avda. Diagonal 645, 08028, Barcelona, Spain

${ }^{\circledR}$ Tel:+34934021873; Fax:+34934021559; Email: simperial@ub.edu

\begin{abstract}
Tuberculosis is one of the leading infection diseases in humans. Discovering new treatments for this disease is urgent, especially due to the emergence of multiple drug resistant organisms and to reduce the total duration of the current treatments. In Mycobacterium tuberculosis the synthesis of isoprenoids has been reported as an interesting pathway to target and especial attention has been focused on the methylerythritol phosphate (MEP) pathway comprising the early steps of biosynthesis. In this context we have studied the enzyme 2C-methyl-Derythritol-4-phosphate cytidylyltransferase (CMS), the third enzyme of the MEP pathway, since so far there is no resolved structure of this protein in M. tuberculosis, and its use as a drug target is therefore seriously limited. We have performed a homology modeling of the $M$. tuberculosis CMS to provide a reliable model to be used in structure-based drug design. After evaluating the quality of the model, we fully studied the catalytic site and the dimerization interface of the model, suggesting the most important sites (conserved and non-conserved) useful for drug discovery and mutagenesis studies. We found that the metal coordination of CDP-methylerythritol is substantially different in the M. tuberculosis CMS with respect to the E. coli variant, corroborating that the former is able to utilize several metal ions for catalysis.
\end{abstract}


Moreover, we propose that electrostatic interactions could explain the higher affinity of the MEP substrate compared with the CTP substrate in the M. tuberculosis enzyme as reported previously.

Keywords 2C-methyl-D-erythritol-4-phosphate cytidylyltransferase (CMS) $\cdot$ Homology modeling $\cdot$ Molecular dynamics $\cdot$ Drug design $\cdot$ Tuberculosis 


\section{Introduction}

Tuberculosis (TB), caused by Mycobacterium tuberculosis, remains the leading infectious disease to humans. It accounts for approximately 8 million new cases worldwide and an estimated 2 million deaths annually [1].

The standard first-line treatment against active TB is based on the administration of the drugs rifampicin, isoniazid, pyrizinamide, and ethambutol given in combination over six to nine months. The combinations are very important to prevent the emergence of multiple drug resistant (MDR) organisms, which can lead to an ineffective treatment [2].

There are three basic factors involved in the development of new tuberculosis drugs; to reduce the total duration of treatment, to improve the MDR TB and to provide more effective treatments of latent tuberculosis infection [3-4].

In Mycobacterium tuberculosis the synthesis of isoprenoids is an important step in cell wall construction [5-7] and oxidative phosphorylation [8]. It is therefore reasonable to predict that the enzymes involved in the early steps of biosynthesis of essential isoprenoids provide new and valid drug targets [6].

Despite the diversity of structure and function, all isoprenoids originate from the common five-carbon (C5) building units, isopentenyl diphosphate (IPP) and its isomer, dimethylallyl diphosphate (DMAPP) [9-10]. To date, two distinct pathways have been discovered for the biosynthesis of the two precursors; the mevalonate (MVA) pathway and the methylerythritol phosphate (MEP) pathway. Most bacterial pathogens, including M. tuberculosis, exclusively utilize the MEP pathway whereas the MVA pathway is the only present in humans [11-13].

The MEP pathway is comprised of seven enzymatic steps starting with the condensation of pyruvate and glyceraldehyde-3-phosphate to produce DXP, which is converted to MEP in reactions catalyzed by DXP synthase [14-16] and DXP reductoisomerase (IspC) [17-18], respectively. In the third step of the pathway, MEP reacts with CTP to produce CDPmethylerythritol and pyrophosphate in a reaction catalyzed by the enzyme 2C-methyl-Derythritol-4-phosphate cytidylyltransferase (EC 2.7.7.60; CMS) [19-22]. Not many inhibitors have been described for the enzymes of the MEP pathway [13]. A paradigmatic example is fosmidomycin, an antibiotic that selectively inhibits DXP reductoisomerase [17-18] and that has proven useful against malaria [12]. 
CMS from E. coli and other bacteria including $M$. tuberculosis have been obtained in recombinant form [19-25]. The steady-state kinetic properties of CMS from E. coli [22], from Streptomyces coelicolor [23] and from M. tuberculosis have been reported [24-25].

X-ray structure studies have been carried out with the bacterial enzymes from E. coli [21], Thermus thermophilus [26] and Thermotoga maritima [27], over others, but so far no crystal structure is yet available for CMS from M. tuberculosis. Moreover, only a weak inhibitor of CMS (erythritol-4-phosphate) has been described [28], but as this compound is a desmethyl analogue of the natural cofactor, it does not provide any structural clue to design new active compounds for different binding sites. Structural analysis showed that CMS organizes as a homodimer, each enzyme subunit being primarily a single domain $\alpha / \beta$ structure constructed around a seven-stranded twisted $\beta$-sheet, into which is inserted an extended $\beta$-arm. Two arms associate to help form a dimer interface that involves numerous inter-subunit hydrogen bonds and several salt bridges. The enzyme active site is created at this interface by seven polypeptide segments, six from one subunit and one from the partner.

Amino acids which are involved in formation of the substrate binding site and the catalytic active site have been identified. A highly basic active site binds and orients the four phosphate groups present in the substrates, and in particular two lysine residues (Lys 27 and Lys 213 in E. coli CMS) are key to the stabilization of the pentavalent transition state generated following an in-line nucleophilic attack by the MEP phosphate on the alpha phosphate of CTP. A complex network of hydrogen bonds positions MEP so that catalysis can occur.

The reaction catalyzed by CMS, like the rest of the reactions involved in the MEP pathway, represents an excellent potential target site for chemotherapy against $M$. tuberculosis. The properties of the enzyme would provide important information for the design of novel antitubercular agents and the differences observed with the CMS from other bacteria would be essential to increase the specificity of these agents for M. tuberculosis.

M. tuberculosis CMS (the Rv3582c gene product) has approximately $32 \%$ identity with $E$. coli CMS and conserved amino acids are involved in formation of the substrate binding site and the catalytic active site. However, some differences have been reported, for instance the amino acid corresponding to S88 of the E. coli enzyme active site has been changed to a threonine in $R v 3582 c$. Recombinant $M$. tuberculosis CMS is active over a relatively broad $\mathrm{pH}$ range (6.0-9.0) [24-25] as was previously reported for the E. coli CMS [22]. A divalent cation, such as $\mathrm{Mg}^{2+}$ or $\mathrm{Mn}^{2+}$, is absolutely required for this cytidylyltransferase activity. $\mathrm{Zn}^{2+}$ supports the M. tuberculosis CMS activity [25]. However, E. coli CMS activity was not 
supported by $\mathrm{Zn}^{2+}$ suggesting a subtle difference in the metal coordination [19, 25]. $M$. tuberculosis CMS showed a high degree of specificity for CTP as a substrate [24-25], unlike other nucleotide 5-phosphates (GTP, UTP, TTP, or ATP).

Due to the rapid spread of multidrug resistant TB strains against all major antituberculosis drugs, there is an urgent need for TB drugs with fewer toxic side effects and improved pharmacokinetics properties which would allow to reduce the total duration of treatment. In this scenario, a structure of the enzyme from M. tuberculosis would be desirable in order to work in structure-based drug design. We report here, the first model of CMS from $M$. tuberculosis, using the 3D structure of the E. coli enzyme as a template, performing homology modeling and refining it by molecular dynamics simulations. Homology modeling is a general strategy to obtain a protein structure when no crystal structure is available, and it has been applied to several protein models with interesting results, including M. tuberculosis DXP reductoisomerase, the second enzyme of the MEP pathway [29]. Moreover, this methodology can suggest mutagenesis studies and useful sites for drug discovery [30].

Our molecular dynamics simulation is used to analyze the energetics of the whole proteinprotein interacting surface as well as the binding site of CDP-methylerythritol, identifying the role of conserved residues between the $E$. coli and the $M$. tuberculosis variants. In light with our study, electrostatic interactions could explain the higher affinity of MEP compared to CTP for binding as reported previously in the M. tuberculosis enzyme [24]. Moreover, a fundamental difference in the metal ion coordination between the M. tuberculosis model and the $E$. coli enzyme is also addressed. Non-conserved regions and identified channels were also analyzed to suggest new binding sites to develop selective inhibitors.

\section{Methods}

\section{Construction of the M. tuberculosis CMS}

Homology modeling was performed using the MODELLER 8v2 program [31] which is based on comparative protein structure by satisfaction of spatial restraints [32-33]. Several X-ray structures of CMS are appropriate and can be used as 3D templates: the E. coli variant (pdb codes 1INI and 1I52 [21]), the Thermotoga maritima variant (pdb code 1VPA [27]), the Thermus termophilus variant (pdb code 2PX7 [26]) and other possible templates including those of the bifunctional IspDF from Campylobacter jejuni (pdb codes 1W55 and 1W57 
[34]), from the Listeria monocytogenes (pdb code 3F1C [35]) and those from Streptococcus pneumoniae (pdb codes 2VSH and 2VSI [36]). We selected the E. coli variant 1INI, which is the solved structure containing the CDP-methylerithritol, because this variant has been exhaustively studied in terms of mutagenesis data and reaction kinetics. The other possible templates, were considered inappropriate for presenting functional differences, less sequence identity or for not containing the complex with CDP-methylerithritol.

Fig. 1 shows the sequence alignment between the $E$. coli and $M$. tuberculosis variants, with a sequence identity of $32 \%$ and presenting only four non-close gaps (only two in secondary structure elements). This alignment was performed using the ALIGN automatic server [37] using default parameters of scoring. A multiple sequence alignment including eight variants was examined [24] and no important differences were found with respect to our pairwise alignment.

Moreover, we carried out a superimposition of the six possible species using the NeedlemanWunsch algorithm, including secondary structure score as appears in the Chimera software [38], to determine if the election of only one of these templates would introduce some bias into the M. tuberculosis CMS homology model. As shown in Fig. 2A, the topology and 3D structures of the templates are highly similar, revealing that selecting more than one template would be a redundant work in this system. Backbone RMS deviations were $2.4 \AA$ for 216 atom pairs between the E. coli and the Thermotoga maritima variants, $2.8 \AA$ for 202 atom pairs between the E. coli and the Thermus thermophilus variants, $5.8 \AA$ for 204 atom pairs between the E. coli and the Campylobaster jejuni variants, $4.5 \AA$ for 215 atom pairs between the E. coli and the Listeria monocytogenes variants and $6.1 \AA$ for 211 pairs between the E. coli and the Streptococcus pneumoniae variants.

It is worth noting that sequence identity among these templates ranges within 25 to $32 \%$. Therefore, despite the low sequence identity, the 3D structures of CMS are very well conserved among different species. M. tuberculosis CMS should not be an exception and for this reason, the sequence identity between this variant and the selected template seemed adequate to perform homology modeling, expecting to obtain also a very similar overall structure.

The MODELLER program was applied to generate thirty satisfactory models for each monomer of $M$. tuberculosis CMS. The model with the lowest energy and the lowest restraint violation was selected to construct the homodimeric system. The STRIDE software [39] was used to predict secondary elements in both the E. coli and the best MODELLER model of $M$. 
tuberculosis CMS (Fig. 1). The secondary structure of the model was similar to the E. coli protein, thus confirming the quality of the homology modeling. As regards the tertiary structure, Fig. 2B shows the best M. tuberculosis model selected superimposed over the crystal structure of E. coli CMS. This figure also shows the reaction product CDPmethylerythritol, placed in both monomers of the protein and superimposed between the $E$. coli and the M. tuberculosis variants. This superimposition was performed using also the Needleman-Wunsch algorithm, including secondary structure score, as appears in the Chimera software [38]. The tertiary structure of both proteins was also very similar, providing a good starting point from which to refine the structure by molecular dynamics. To reveal the differences among the generated models, the five MODELLER models with the best scores for each monomer of $M$. tuberculosis CMS were compared. The maximum backbone RMS deviation was $2.26 \AA$. The monomer with the best score was selected to construct the enzyme homodimer (shown in Fig. 2B) taking into account that structures with a maximum deviation of $2.26 \AA$ should be found during an usual molecular dynamics simulation. Thus, performing different molecular dynamics with other worse scored MODELLER models was not considered to contribute to obtain substantially different $M$. Tuberculosis structures.

The M. tuberculosis CMS dimer was constructed by imposing the same symmetry as that of the E. coli CMS homodimer.

In the second step, hydrogen atoms were added to the structure by using the LEAP program of the AMBER-7 package [40].

The model was completed with the addition of two CDP-methylerythritol molecules (superimposing their position and conformation from the E. coli protein) and the two structural magnesium cations coordinated to the diphosphate fragment of CDPmethylerythritol.

The AMBER force field parameters of CDP-methylerythritol were calculated with the General Amber Force Field (GAFF) program [41] using a charge population derived from an adjustment to electrostatic potential (RESP) implemented in the GAUSSIAN package [42].

Finally, a cubic box of approximately 22,000 TIP3P water molecules was added to the system. Molecular dynamics was performed in an explicit solvent model, using periodic boundary conditions within the particle mesh Ewald (PME) context [43]. The system was neutralized by adding an appropriate number of sodium counterions.

All calculations were carried out at the molecular mechanics level using the Cornell et al. force field [44] with the AMBER-7 suite of programs [40]. 


\section{Minimization and molecular dynamics}

The M. tuberculosis CMS homodimer was energy-minimized, using the steepest descent algorithm, to remove bad contacts derived from the homology modeling and to achieve a good starting structure with which to perform molecular dynamics.

In the first step, of 1,000 iterations, only water and counterion molecules were allowed to move. In the second step, of 1,500 iterations, water, counterions and protein side chains were allowed to move. Finally 50,000 iterations were calculated with the restriction-free system up to an energy gradient lower than $1 \mathrm{kcal} \mathrm{mol}^{-1}$.

The minimized structure was heated by coupling the system to a thermal bath at $300 \mathrm{~K}$ using Berendsen's algorithm [45] and at a constant rate of $30 \mathrm{~K} / 10 \mathrm{ps}$; all atoms except water and counterions were maintained frozen. Time step was set to $2 \mathrm{fs}$ and the list of nearest neighbor atoms was updated every 15 ps. A cut-off distance of $9 \AA$ was chosen to compute non-bonded interactions and the SHAKE [46] algorithm was imposed in order to constrain all bonds involving hydrogen atoms.

Once the system was heated a second step of 40 ps at constant pressure was calculated to increase the density of the system. Restrictions on solute main chains and cofactors were then relaxed gradually until the system was free. Finally, $7.6 \mathrm{~ns}$ of unrestrained molecular dynamics (MD) were calculated within the NVT ensemble at a constant temperature of $300 \mathrm{~K}$.

\section{Binding free energy calculation}

The Molecular Mechanics Generalized Born Surface Area (MMGBSA) protocol [47] was applied to estimate the binding free energy of CDP-methylerythritol in complex with the $M$. tuberculosis CMS model as well as to evaluate the energetics of the dimer interface. This protocol was used within the one-trajectory approximation, and computes the binding free energy as follows:

$$
\begin{aligned}
& \Delta \mathrm{G}_{\text {binding }}=\mathrm{G}_{\text {complex }}-\left(\mathrm{G}_{\text {receptor }}+\mathrm{G}_{\text {ligand }}\right) \\
& \mathrm{G}=\mathrm{E}_{\mathrm{MM}}+\mathrm{G}_{\text {Polar Solv }}+\mathrm{G}_{\text {Non-Polar Solv }}-\mathrm{TS} \\
& \mathrm{E}_{\mathrm{MM}}=\mathrm{E}_{\mathrm{int}}+\mathrm{E}_{\mathrm{vdw}}+\mathrm{E}_{\text {ele }} \\
& G_{\text {Non-Polar Solv }}=a S A S A
\end{aligned}
$$


Where $\mathrm{E}_{\mathrm{MM}}$ denotes bond, angle and dihedral force field energies, and $\mathrm{E}_{\mathrm{vdw}}$ and $\mathrm{E}_{\text {ele }}$ are the van der Waals and electrostatic energies. An external dielectric constant of 80 was set to treat the system with implicit solvation. The Generalized Born methodology was applied to solve the polar contribution to solvation $\left(\mathrm{G}_{\text {Polar Solv }}\right)$, using the parameterization of Tsui and Case [48].

Finally, $\mathrm{G}_{\mathrm{Non}-\text { Polar Solv }}$ is the non-polar contribution to solvation, related linearly with the solvent accessible surface area (SASA), while $a$ takes the value of $0.0072 \mathrm{kcal} \mathrm{mol}^{-1} \AA^{-2}$.

SASA was computed by means of the Linear Combination of Pairwise Overlap (LCPO) method [49.] Entropic effects (TS) were computed through a normal mode analysis using the nmode module of AMBER-7. The Minimization step before normal mode analysis was carried out up to an energy gradient of $10^{-4} \mathrm{kcal} \mathrm{mol}^{-1}$. It is important to note that, due to limitations of the method, both intrinsic and based on the MD simulation [50], the absolute numerical values of the binding free energy are not relevant for these studies, being in general larger than the experimental data [51]. Only their relative values are important and informative, and consequently, the numerical value of the total binding free energy could not be close to the experimental data.

MMGBSA was applied to 100 equidistant snapshots extracted from the production time, except for the entropic contribution, which is computationally intensive, and therefore is usually based only on a few snapshots. For this contribution, 10 snapshots were extracted by limiting the system only to those residues located at a distance of $9 \AA$ from the CDPmethylerythritol molecules.

\section{Results}

\section{Assessment of the M. tuberculosis CMS model}

The total energy of the system versus time was examined in order to verify the stability of the model; last $2 \mathrm{~ns}$, from a total of $7.6 \mathrm{~ns}$, were considered as the production time, and used to extract structural and energetic results. In addition, the average backbone RMS deviation of the full system was $4.3 \AA$, which can be considered as a measure of the quality and stability of the model. This value decreased to $3.9 \AA$ not accounting the first 10 residues of both ends. 
Energy and RMS convergence was achieved after more than 5 ns of molecular dynamics, due to the slow conformational changes of side chains together with the low sequence identity of the two variants. As can be seen, a long molecular dynamics is necessary to improve the initial MODELLER structure, and in general, to refine a homology model with low sequence similarity.

As regards CDP-methylerythritol, the average all-atom RMS deviation of the first CDPmethylerythritol molecule with respect to the conformation in the E. coli variant was $2.4 \AA$ (similar results were obtained for the second CDP-methylerythritol molecule). This is also a small deviation, in agreement with a good binding site description.

As a final test of the quality of the model, four structure evaluation programs were used; PROCHECK [52], Verify-3D [53], ProSA [54] and ERRAT [55]. The former was applied to quantify the residues in available zones of the Ramachandran plot, selecting the MODELLER model of M. tuberculosis CMS. Thus, $88.3 \%$ of residues were located in the most favored zones, $9.6 \%$ in allowed regions, $1 \%$ in generously allowed regions, and $1 \%$ in disallowed regions. Similar results were found in the E. coli template: $81.8 \%$ of residues in the most favored zones, $17.7 \%$ in allowed regions, no residues in generously allowed regions, and $0.5 \%$ of residues in disallowed regions.

Verify-3D uses a score function to assess the quality of the model. Fig. 3 shows the Verify-3D profile for the complete homodimer, selecting the initial model and the final molecular dynamics structure; residues with a score over 0.2 should be considered reliable. Thus, $51.6 \%$ of residues have a score over 0.2 in the initial model and $63.6 \%$ in the last molecular dynamics structure since the structure was substantially improved, specifically in the sequence 202-220. This fragment corresponds with a structure with no secondary elements in M. tuberculosis CMS (Fig. 1) and therefore difficult to model, nevertheless the subsequent molecular dynamics improved this fragment of the protein as indicated by this score.

Additionally, Fig. 4 shows the energy profiles for the MODELLER model (middle) and for the final molecular dynamics structure (bottom) using the ProSA score and compared with the experimental structure of E. coli CMS (top). This program evaluates the energy of the structure using a distance-based pair potential. Residues with negative ProSA energies confirm the reliability of the model. As can be seen, the homology model shows a similar ProSa profile with respect to the template, and additionally the molecular dynamics decreased the positive peaks of the middle zone to better compare this profile with the E. coli structure. 
Finally, an overall quality factor of $50.2 \%$ for the MODELLER structure was obtained using the ERRAT function, which is based on statistics of non-bonded interactions. This score was highly improved in the molecular dynamics structure, giving an overall quality factor of 79.3 and $86.6 \%$ for monomer one and two, respectively. These results are very similar to the overall quality factor obtained for the E. coli template, which was $89.2 \%$.

Main differences before and after molecular dynamics, as indicated by these scoring functions, were found into loops of M. tuberculosis CMS. Particularly, in loops between residues 14-26, 147-152 and 202-220. Fig. 5A shows the positions of these three loops in the MODELLER structure, and Fig. 5B focuses on the short alpha helix obtained in loop 14-26 after molecular dynamics, corresponding with a short helix found in the template structure (Fig.1). This was the most remarkable structural change of the backbone during the simulation.

\section{Role of conserved residues in M. tuberculosis CMS}

Shi et al. (2007) [24] performed an accurate study of E. coli CMS mutants and identified invariant residues. A role in catalytic activity was suggested for these residues, and two signature motifs of CMS were found. We have used our homology model of M. tuberculosis CMS to suggest the role of some of these conserved residues. In addition, this analysis is also utilized to shed light into the experimental affinities of the MEP and CTP substrates reported by Shi et al. [24] in M. tuberculosis CMS (Fig. 6). The MEP molecule, being smaller than CTP and maintaining therefore less contacts with the protein, has 2-fold more affinity for CMS $(43 \mu \mathrm{M}$ vs. $92 \mu \mathrm{M})$.

The similarity between CMS from different species is very high in the binding site which is adapted to high-charged substrates, such as CTP. Molecules able to interact in this zone should present a diphosphate or analogue group, nevertheless some authors described nonphosphate inhibitors to target the isoprenoid biosynthesis [56]. An MMGBSA 'per residue' protocol [57] was performed to identify the residues that stabilize CDP-methylerythritol. The interaction energies of residues of the whole protein was calculated with respect to the CDPmethylerythritol molecule present in the first monomer, averaged over 100 snapshots of the converged molecular dynamics. Table 1 marks the results of this analysis, showing the $\Delta \mathrm{GB}_{\mathrm{TOT}}$ contribution (which includes van der Waals and electrostatic interactions, polar solvation contribution and non-polar solvation contribution) of the CMS residues that offered the main contributions to binding. Important residues of M. tuberculosis CMS were P13, A14, 
A15 and G16 which form an invariant motif among different CMS species, called the glycinerich loop. This cluster of residues contributes with $-7.96 \mathrm{kcal} \mathrm{mol}^{-1}$ to the total binding (Table $1)$.

T86 which corresponds to similar S88 in the E. coli variant is much less important, but was postulated to sequester the CDP-methylerythritol in the E. coli enzyme [24]. Conserved A108 interacts also weakly and similarly to the same residue in E. coli (A107) [22]. R110 and $\mathrm{K} 219$, which also play a role in dimerization, contribute with -8.30 and $-2.46 \mathrm{kcal} \mathrm{mol}^{-1}$, respectively (Table 1). In fact, R110 is the most critical residue for CDP-methylerythritol stabilization and its mutation in the E. coli enzyme (R109A) has been reported to cause a drastic inhibition [22]. Since this residue interacts with a hydroxyl of the MEP moiety of CDP-methylerythritol, we propose that R110 is in part responsible of the higher affinity of the MEP substrate $(43 \mu \mathrm{M})$ compared to CTP $(92 \mu \mathrm{M})$ reported previously in the $M$. tuberculosis protein [24]. As shown in the MMGBSA analysis of Table 1, R110 offers the most energetic stability per residue to the CDP-methylerythritol binding. Additionally, we also decomposed the CDP-methylerythritol into chemical groups and calculated the average van der Waals and electrostatic energies from the force field to shed light into the reported affinities (Fig. 6). The methylerythritol fragment had a van der Waals interaction energy of $-9.14 \mathrm{kcal} \mathrm{mol}^{-1}$, whereas the ribose and the pyrimidine groups showed a van der Waals interaction of -9.03 and -9.23 kcal $\mathrm{mol}^{-1}$, respectively. Nevertheless, experimental affinities can be correlated taking into account the electrostatic interaction energy too. In this case, the amino group attached at the pyrimidine has an average electrostatic energy of $-5.68 \mathrm{kcal} \mathrm{mol}^{-1}$ and the two hydroxyl groups of the ribose offer $-12.23 \mathrm{kcal} \mathrm{mol}^{-1}$, but the three hydroxyl groups of the methylerythritol maintain a much higher electrostatic interaction energy with the protein, $39.47 \mathrm{kcal} \mathrm{mol}^{-1}$. All in all, although hydrophobic recognition is identified most in the moiety coming from the CTP, the much higher electrostatic energies, that can explain the different affinity of the substrates, are only focused on the hydroxyl groups of the methylerythritol fragment.

Finally, conserved T141 of the second monomer which is also involved in dimerization, interacts with $-1.55 \mathrm{kcal} \mathrm{mol}^{-1}$ (Table 1) being also important for E. coli CMS. Thus, mutation of this residue in E. coli (T140V) was shown to highly decrease enzyme activity [22], presumably by a steric effect. The result of the E. coli CMS mutation T165V [22] is more ambiguous. This residue (also T165 in M. tuberculosis CMS) makes a weak hydrogen bond with the methylerythritol moiety in the second monomer of our model. Nevertheless, this contact is lost in the first subunit but replaced with another hydrogen bond with R110. This 
could be an effect of the protein flexibility that effectively exchanges a hydrogen bond between R110 and T165. Other mutations were also important affecting the E. coli CMS activity: K27A, K27S and K213S. These two residues were postulated to affect the transition state of the reaction by a mechanism of charge stabilization. In our analysis by the MMGBSA protocol no special role for these residues (K27 and K215) in the product stabilization of $M$. tuberculosis CMS was observed. We modeled a system with the reaction product and thus some residues should show different orientations with respect to the reactive or transition state structures. Another possibility is that these two residues are not effectively involved in direct interactions with CDP-methylerythritol as our model suggests. Therefore, we propose to test the effect of the K27A, K27S and K215S mutations on $\mathrm{k}_{\mathrm{cat}} / \mathrm{K}_{\mathrm{m}}$ in M. tuberculosis CMS to shed some light into the role of these two residues. As regards D193, this residue makes an unfavorable contact with CDP-methylerythritol (Table 1) due to its negatively charged side chain which is in close contact with the diphosphate group of CDP-methylerythritol, but this residue is coordinated to the structural magnesium and thus the unfavourable interaction is corrected. This MMGBSA analysis does not take into account this effect. Obviously, the magnesium ion and CDP-methylerythritol make the most important contribution to the total binding free energy for the complexation process (Table 1).

To complement these results, Fig. 7 shows a visual representation of the most important hydrogen bonds between the protein and CDP-methylerythritol being similar in the E. coli variant (residues with van der Waals contacts were not shown for simplicity). The magnesium coordination is also shown. The carbonyl group of P13 forms a hydrogen bond with a hydroxyl group of the ribose moiety, the amino groups of A14, A15 and G16 form three hydrogen bonds with the carbonyl of the pyrimidine base (the former is weak) similarly as described in the E. coli variant. In our model the methyl group of T86 interacts by van der Waals forces with the pyrimidine base, the methyl of A108 interacts in the same manner with the ribose ring, the side chain of R110 interacts by electrostatic forces with the phosphate groups and with a hydroxyl of methylerythritol offering the most important contribution, the side chain of K219 also forms contacts with a hydroxyl group of methylerythritol. Finally, the methyl group of T141 of the second monomer makes a van der Waals contact with the methyl group of methylerythritol. Other residues involved in hydrogen bonds to the product are N82, T84, A109, T217, T218 from the first monomer and D140 from the second. Two strong hydrogen bonds localized in the erythritol fragment are formed with charged residues, D140 and R110, thus explaining the strong electrostatic interaction energy of the hydroxyl groups of this moiety discussed before. 
The structural magnesium ion is stabilized by contacting with two oxygen atoms of the phosphate group of CDP-methylerythritol, with both side chain oxygen atoms and the carbonyl group of D193 and with the side chain hydroxyl of T84 (Fig. 7). The structural magnesium ion located in the second subunit shows a slightly different coordination in the last molecular dynamics structure. Thus, the carbonyl of D193 that makes a contact with magnesium in the first subunit is replaced by the carbonyl of T84 in the second subunit. Both carbonyl fragments of T84 and D193 show a dynamical behaviour throughout the simulation, but the same average coordination is always maintained. We suggest that D193A and T84A mutations should distort the ion coordination and affect the catalytic activity of $M$. tuberculosis CMS. Interestingly, the magnesium ion is not coordinated to any residue of the protein in the crystal E.coli CMS [21] so its coordination is not as tight as shown in our model of M. tuberculosis CMS. This fundamental difference may cause that the M. tuberculosis variant can accept a zinc ion (which has an ionic radius slightly larger than magnesium and so different polarizability) whereas the E. coli variant cannot [19] and is more specific for magnesium. A fundamental difference in metal coordination between the $E$. coli and the $M$. tuberculosis enzymes was early suggested by Eoh et al. [25] based on their affinities for different metal ions. The presented model is able to suggest a structural explanation, involving D193 and T84, for these experimental data.

In order to elucidate the driving force of binding, we estimated the binding free energy of CDP-methylerythritol in complex with the protein, also including the magnesium as part of the receptor as our previous study suggested in a similar metal-coordinated cofactor [57]. Results of this analysis are shown in Fig. 8. The hydrophobic balance upon binding (VDW $\left.+\Delta \mathrm{GB}_{\mathrm{SUR}}\right)$ was approximately three-fold lower than the electrostatic balance (ELE $+\Delta \mathrm{GB})$. According to these results, binding seems to be driven by electrostatics forces; this agrees with our previous MMGBSA studies carried out on similar ligands with the phosphate group [58].

Inclusion of entropy by a normal mode analysis decreased the final energy in $19.27 \mathrm{kcal} \mathrm{mol}^{-}$ ${ }^{1}$, being not decisive for binding.

It is worth to remark here that the final binding free energy is unrealistic because the high electrostatic energy extracted from the force field is not well balanced by the desolvation penalty using the GB approach. Overestimated values of the binding free energy are usually found in literature $[58,51]$ and for this reason MMGBSA is useful to study the relative contributions to binding but must be taken with caution when discussing absolute values. 


\section{Description of the dimerization interface by the MMGBSA protocol}

An MMGBSA 'per residue' protocol [57] was also applied to calculate the binding free energy contribution of all residues of the first monomer with respect to the whole second monomer. In this approach, the most important residues that show the strongest energies, should contribute to the stability of the homodimer. Moreover, small molecules that could bind to these critical residues could be used to disrupt dimerization and to inhibit the protein as Shi et al., 2007 [24] suggested an active homodimer for M. tuberculosis CMS.

This methodology describes not only the interactions 'in vacuo' but also adds the solvation contribution to the final binding free energy. Fig. 9 shows the results of this analysis, plotting the $\Delta \mathrm{GB}_{\mathrm{TOT}}$ contribution (which includes van der Waals and electrostatic interactions, polar solvation contribution and non-polar solvation contribution) versus each important CMS residue of the first monomer and averaged over 100 snapshots extracted from the molecular dynamics. Also included are CDP-methylerythritol and the structural magnesium. It can be seen that binding between subunits is driven by small clusters or "hot spots" that play the major role in protein-protein recognition. Eleven residues that contribute with the highest binding energies: T141, I142, D146, R161, V163, D193, A194, S195, I200, K219 and I228, were identified. All these residues are located in the middle and C-terminal regions of the protein. The first five residues are located within the extended $\beta$-arm domain and contribute with the strongest interactions, while the rest are located within the globular domain. We suggest that T141A and I142A mutations should have important effects on the dimer stability of CMS. T141 also contributes to CDP-methylerythritol stabilization (as discussed before) and therefore its mutation could disrupt the dimer formation and at the same time distort the catalytic site.

It is worth to remark that T141, A194 and S195 are conserved residues and that I142, V163, D193 are similar residues in the E. coli CMS, therefore the dimerization interface is clearly conserved. With respect to CDP-methylerythritol, it contributes slightly $\left(3 \mathrm{kcal} \mathrm{mol}^{-1}\right)$ to the dimer stability (Fig. 9).

\section{Role of non-conserved residues in M. tuberculosis CMS}

Seven zones where the sequence identity between $M$. tuberculosis CMS and the E. coli variant is substantially decreased were identified and analyzed: L21-V25 (0\% identity), D47- 
T53 (14\% identity), R62-I76 (7 \% identity), A111-L117 (0 \% identity), E123-136 (0\% identity), V145-G153 (0\% identity) and L175-Y190 (19\% identity). A potential compound able to interact with these zones would be of great interest for a selective inhibition of $M$. tuberculosis CMS. Some of these non-conserved zones include loops or sequences without secondary structure, and are located entirely in the globular domain except V145-G153 which is in the middle zone of the $\beta$-arm domain. These zones presented high mobility during the molecular dynamics simulation, and docking procedures aimed to find molecules onto them should take into account these flexible structures. Thus, a docking procedure with induced-fit effects should be preferred.

B-factors (Fig. 10) using normal mode analysis were carried out using the elNémo server [59] corroborating the flexibility of these sequences. Interestingly, the most flexible loop was found between residues R20 and K27 that is the one implicated in catalysis in E. coli CMS, as suggested by Richard et al. [21]. The fourth sequence (A111-L117) which is not flexible, is close to the catalytic site and should be a potential new site for drug development, focusing on selectivity. On the other hand, the R62-I76 sequence is enclosed in the channel 8 discussed in the next part of the manuscript, so it seems also suitable for docking studies to find selective drug candidates.

\section{Identification of channels in M. tuberculosis CMS}

The MolAxis algorithm [60] which is based on the $\alpha$-shape theory and on the geometrical concept of the median axis was used to identify channels in the refined model of $M$. tuberculosis CMS. Thus, identified channels that connect inside cavities with the outside of the protein can be used as reference sites for drug discovery. Table 2 shows 10 identified channels in the homodimer model, and the gating residues (or "bottleneck" residues) involved in these channels. Interestingly, all channels, except channels 5, 7 and 8, are composed of residues contributed by both monomers. All channels are well conserved except channels 7 and 10 .

Fig. 11 shows these gating residues highlighted in the complete homodimer structure. As can be seen, gating residues of channels 6 and 10 (white and black, respectively) are close to the catalytic site, and therefore any compound that could bind to these proposed sites would probably inhibit M. tuberculosis CMS allosterically. In this line, the inset in Fig. 11 shows a detailed view of the best scored channel and its gating residues. L220 and L222 of the first monomer constitute a hydrophobic cluster, while carbonyl groups of P211 and K215 of the 
second monomer make a polar cluster. Interestingly, the proximity of this channel to the catalytic pocket suggests that this site is the entrance of the natural substrates of CMS. The analysis shown before in Fig. 10 also indicated that this sequence is highly rigid accounting to the fine selectivity of the enzyme for its substrates. Therefore, the gating residues of this channel could be used as a reference site to carry out a virtual screening, focused on finding new inhibitors by blockage of the CMS substrates.

\section{Conclusions}

Tuberculosis remains one of the leading infectious diseases to humans and the isoprenoid biosynthesis in $M$. tuberculosis has been validated as a new target pathway for the future development of specific drugs against tuberculosis. The third enzyme involved in this pathway is 2C-methyl-D-erythritol-4-phosphate cytidylyltransferase (CMS). Since no structure of this enzyme from $M$. tuberculosis has been yet reported, we presented a homology model refined through molecular dynamics using the resolved E. coli CMS crystal structure as template. We analyzed the quality of the model in detail, in terms of secondary and tertiary structure, as well as the energetics of the model applying different assessment programs and the MMGBSA protocol. We concluded that the reported M. tuberculosis homology model seems reliable in light of the scoring functions applied and could be used in drug design strategies. The model was further analyzed to address the role of conserved and non-conserved residues. The formers were located in the substrate binding site and in the dimerization interface, whereas non-conserved residues were localized in loops with high mobility during the simulation. Overall, our model provided the main insights: i) the most important residues for the dimer stability were conserved T141 and I142; ii) the higher affinity of the MEP substrate compared with the CTP substrate in M. tuberculosis CMS could be attributed to the hydrogen bonding with conserved R101, and to the much higher electrostatic interactions of the hydroxyl groups of the MEP moiety; iii) there is a fundamental difference in the metal binding between the $M$. tuberculosis model and the $E$. coli structure, as the metal ion was coordinated by using the residues D193 and T84 in our reported model, that were absent in the crystal structure of E. coli CMS. This finding should corroborate with the ability of $M$. tuberculosis CMS to support several metal ions for catalysis, whereas the E. Coli variant is more specific; iv) the identified channel with the gating residues L220, L222, K215 and R211 seems appropriate to carry out a virtual 
screening protocol for identifying molecules able to block the entrance of the natural substrates of the protein.

In the absence of the crystal structure of the third enzyme of the MEP pathway in the $M$. tuberculosis organism, we have presented an accurate model to be used in future drug design protocols.

\section{Acknowledgments}

The Spanish Ministry of Science and Technology supported this work through the projects CTQ2006-06588/BQU and BIO2002-04419-C02-02. The 'Generalitat de Catalunya' also supported this work through the grants 2005SGR00914 and 2009SGR1308. We are also grateful to the 'Departament d'Universitat, Recerca i Societat de la informació de la Generalitat de Catalunya i del Fons Social Europeu'. 


\section{References}

1 Global tuberculosis control: surveillance, planning, financing (2007) (WHO/HTM/TB/2007376)

2. Vinícius M, de Souza N (2006) Promising drugs against tuberculosis. Recent Patents Anti-Infect Drug Disc 1:33-44

3. O’Brien RJ, Nunn PP (2001) The need for new drugs against tuberculosis. Obstacles, opportunities and next steps. Am J Respir Crit Care Med 162:1055-1058

4. Mukherjee JS, Rich ML, Socci AR, Joseph JK, Viru FA, Shin SS, Furin JJ, Becerra MC, Barry DJ, Kim JY, Bayona J, Farmer P, Fawzi MCS, Seung KJ (2004) Programs and principles in treatment of multidrug-resistant tuberculosis. Lancet 363:474-481

5. Wolucka BA, McNeil MR, Hoffmann E, Chojnacki T, Brennan PJ (1994) Recognition of the lipid intermediate for arabinogalactan/arabinomannan biosynthesis and its relation to the mode of action of ethambutol on mycobacteria. J Biol Chem 269: 23328-23335

6. Mikusova K, Mikus M, Besra GS, Hancock I, Brennan PJ (1996) Biosynthesis of the linkage region of the mycobacteria cell wall. region J Biol Chem 271:7820-7828

7. Mahapatra S, Yagi T, Belisle JT, Espinosa BJ, Hill PJ, McNeil MR, Brennan PJ, Crick DC (2005) Mycobacterial lipid II is composed of a complex mixture of modified muramyl and peptide moieties linked to decaprenyl phosphate. J Bacteriol 187:2747-2757

8. Collins MD, Pirouz T, Goodfellow M, Minnikin DE (1977) Distribution of menaquinones in actinomycetes and corynebacteria. J Gen Microbiol 100:221-230

9. Sacchettini JC, Poulter CD (1997) Creating isoprenoid diversity. Science 277:1788-1789

10. Bochar DA, Freisen JA, Stauffacher CV, Rodwell VW (1999) In: Comprehensive Natural Products Chemistry. Pergamon Press, Oxford

11. Testa CA, Brown MJ (2003) The methylerythritol phosphate pathway and its significance as a novel drug target. Curr Pharm Biotechnol 4:248-259

12. Rodríguez-Concepcion M (2004) The MEP pathway: a new target for the development of herbicides, antibiotics and antimalarial drugs. Curr Pharm Des 10:2391-2400

13. Eoh H, Brennan PJ, Crick DC (2008) The Mycobacterium tuberculosis MEP (2C-methylD-erythritol 4-phosphate) pathway as a new drug target. Tuberculosis 89:1-11

14. Sprenger GA, Schörken U, Wiegert T, Grolle S, De Graaf AA, Taylor SV, Begley TP, Bringer-Meyer S, Sahm H (1997) Identification of a thiamin-dependent synthase Escherichia coli required for the formation of 1-deoxy-Dxylulose 5-phosphate precursor to isoprenoids, thiamin and piridoxol. Proc Natl Acad Sci USA 94:12857-12862 
15. Lois LM, Campos N, Putra SR, Danielsen K, Rohmer M, Boronat A (1998) Cloning and characterization of a gene from Escherichia coli encoding a transketolase-like enzyme that catalyzes the synthesis of D-1-deoxyxylulose 5-phosphate, a common precursor for isoprenoid, thiamin, and pyridoxol biosynthesis. Proc Natl Acad Sci USA 95:2105-2110

16. Lange BM, Wildung MR, McCaskill D, Croteau R (1998) A family of transketolases that directs isoprenoid biosynthesis via a mevalonate-independent pathway. Proc Natl Acad Sci USA 95:2100-2104

17. Kuzuyama T, Takahashi S, Watanabe H, Seto H (1998) Direct Formation of 2-C-MethylD-Erythritol 4-Phosphate from 1-Deoxy-D-Xylulose 5-Phosphate by 1-Deoxy-DXylulose 5-Phosphate Reductoisomerase, A New Enzyme in the Non-Mevalonate pathway to Isopentenyl Diphosphate. Tetrahedron Lett 39:4509-4512

18. Proteau PJ (2004) 1-Deoxy-D-xylulose 5-phosphate reductoisomerase: an overview. Bioorg Chem 32:483-493

19. Rohdich F, Wungsintaweekul J, Fellermeier M, Sagner S, Herz S, Kis K, Eisenreich W, Bacher A, Zenk MH (1996) Cytidine 5ф-triphosphate-dependent biosynthesis of isoprenoids: YgbP protein of Escherichia coli catalyzes the formation of 4diphosphocytidyl-2-C-methylerythritol. Proc Natl Acad Sci USA 96:11758-11763

20. Kuzuyama T, Takagi M, Kaneda K, Dairi T, Seto H (2000) Formation of 4-(cytidine $5 \varnothing-$ diphospho)-2-C-methyl-D-erythritol from 2-C-methyl-D-erythritol 4-phosphate by 2-Cmethyl-D-erythritol4-phosphate cytidylyltransferase, a new enzyme in the nonmevalonate pathway. Tetrahedron Lett 41:703-706

21. Richard SB, Bowman ME, Kwiatkowski W, Kang I, Chow C, Lillo AM, Cane DE, Noel JP (2001) Structure of 4-diphosphocytidyl-2-C-methylerythritol synthetase involved in mevalonate-independent isoprenoid biosynthesis. Nature Struct Biol 8:641-648

22. Richard S, Lillo A, Tetzlaff C, Bowman M, Noel J, Cane D (2004) Kinetic analysis of Escherichia coli 2-C-Methyl-D-erythritol-4-phosphate Cytidyltransferase, wild type and mutants, reveals roles of active site amino acids. Biochemistry 43:12189-12197

23. Cane DE, Chow C, Lillo A, Kang I (2001) Molecular cloning, expression and characterization of the first three genes in the mevalonate-independent isoprenoid pathway in Streptomyces coelicolor. Bioorg Med Chem 9:1467-1477

24. Shi W, Feng J, Zhang M, Lai X, Xu S, Zhang X, Wang H (2007) Biosynthesis of isoprenoids: characterization of a functionally active recombinant 2-C-methyl-Derythritol 4-phosphate cytidyltransferase (IspD) from Mycobacterium tuberculosis H37Rv. Biochem Mol Biol 40:911-920 
25. Eoh H, Brown AC, Buetow L, Hunter WH, Parish T, Kaur D, Brennan PJ, Crick DC (2007) Characterization of the Mycobacterium tuberculosis 4-diphosphocytidyl-2-Cmethyl-D-erythritol synthase: potential for drug development. J Bacteriology 189:89228927

26. Chen L, Tsukuda M, Ebihara A, Shinkai A, Kuramitsu S, Yokoyama S, Chen LQ, Liu ZJ, Lee D, Chang SH, Nguyen D, Rose JP, Wang BC. Crystal structure of 2-C-methyl-Derythritol 4-phosphate cytidylyltransferase from Thermus thermophilus HB8.To be published.

27. Joint Center for Structural Genomics (JCSG). Joint Center for Structural Genomics (JCSG) Crystal structure of 2-C-methyl-D-erythritol 4-phosphate cytidylyltransferase (TM1393) from Thermotoga maritima at 2.67 A resolution. To be published.

28. Lillo AM, Tetzlaff CN, Sangari FJ, Cane DE (2003) Functional expression and characterization of EryA, the erythritol kinase of Brucella abortus, and enzymatic synthesis of L-erythritol-4-phosphate. Bioorg Med Chem Lett 13:737-739

29. Singh N, Avery MA, McCurdy CR (2007) Towards Mycobacterium tuberculosis DXR inhibitor design: homology modeling and molecular dynamics simulation. J Comput Aided Mol Des 21:511-522

30. Hillisch A, Pineda LF, Hilgenfeld R (2004) Utility of homology models in the drug discovery process. Drug Discov Today 9:659-669

31. Eswar N, Mari-Renom MA, Webb B, Madhusudhan MS, Eramian D, Shen M, Pieper U, Sali A (2006) Comparative protein structure modeling with MODELLER. Current protocols in Bioinformatics. John Wiley \& Sons, Inc, Supplement 15, 5.6.1-5.6.30, 200

32. Sali A, Blundell TL (1993) Comparative protein modelling by satisfaction of spatial restraints. J Mol Biol 234:779-815

33. Fiser A, Do RK, Sali A (2000) Modeling of loops in protein structures. Protein Sci 9:1753-1773

34. Gabrielsen M, Bond CS, Hallyburton I, Hecht S, Bacher A, Einsenreich W, Rohdich F, Hunter WN (2004) Structure of the bifunctional ISPDF from Campylobacter jejuni. J Biol Chem 279:52753-52761

35. Patskovsky Y, Ho J, Toro R, Gilmore M, Miller S, Groshong C, Sauder MJ, Burley SK, Almo SC. Crystal structure of 2-C-methyl-D-erythritol-4-phosphate cytidiltransferase from Listeria monocytogenes. To be published. 
36. Baur S, Marles-Wright J, Buckenmaier S, Lewis RJ, Vollmer W (2009) Synthesis of CDP-activated ribitol for teichoic acid precursors in Streptococcus pneumoniae. $\mathbf{J}$ Bacteriol 191:1200-1210

37. http://xylian.igh.cnrs.fr/bin/align-guess.cgi

38. Pettersen EF, Goddard TD, Huang CC, Couch GS, Greenblatt DM, Meng EC, Ferrin TE (2004) UCSF Chimera-a visualization system for exploratory research and analysis. J Comput Chem 25:1605-1612

39. Heinig M, Frishman D (2004) STRIDE: a web server for seconday structure assigment from known atomic coordinates of proteins. Nucleic Acids Res 32:W500-W502

40. Case DA, Pearlman DA, Caldwell JW, Cheathan III TE, Wang J, Ross WS, Simmerling CL, Darden TD, Merz KM, Stanton RV, Cheng AL, Vincent JJ, Crowley M, Tsui V, Gohlke H, Radmer RJ, Duan Y, Pitera J, Massova I, Seibel GL, Sligh UC, Weiner PK, Kollman PA (2002) AMBER 7. Univ California, San Francisco

41. Wang J, Wolf RM, Caldwell JW, Kollman PA, Case DA (2004) Development and testing of a general amber force field. J Comput Chem 25:1157-1174

42. Frisch MJ, Trucks GW, Schlegel HB, Scuseria GE, Robb MA, Cheeseman JR, Zakrzewski VG, Montgomery JA Jr, Stratmann RE, Burant JC, Dapprich S, Millam JM, Daniels AD, Kudin KN, Strain MC, Farkas O, Tomasi J, Barone V, Cossi M, Cammi R, Mennucci B, Pomelli C, Adamo C, Clifford S, Ochterski J, Petersson GA, Ayala PY, Cui Q, Morokuma K, Malick DK, Rabuck AD, Raghavachari K, Foresman JB, Cioslowski J, Ortiz JV, Baboul AG, Stefanov BB, Liu G, Liashenko A, Piskorz P, Komaromi I, Gomperts R, Martin RL, Fox DJ, Keith T, Al-Laham MA, Peng CY, Nanayakkara A, Gonzalez C, Challacombe M, Gill PMW, Johnson B, Chen W, Wong MW, Andres JL, Head-Gordon M, Replogle ES, Pople JA (1998) Gaussian 98. Revision A.6. Gaussian, Inc, Pittsburgh PA

43. Darden T, York D, Pedersen L (1993) Particle mesh Ewald: an W $\log (\mathrm{N})$ method for Ewald sums in large systems. J Chem Phys 98:10089-10092

44. Cornell WD, Cieplak P, Bayly CI, Goud IR, Mertz KM Jr, Ferguson DM, Spellmeyer DC, Fox T, Caldwell JW, Kollman PA (1995) A second generation of force fields for the simulation of proteins, nucleic acids and organic molecules. J Am Chem Soc 117:51795197

45. Berendsen HJC, Postman JPM, Van Gunsteren WF, DiNola A, Haak JA (1984) Molecular dynamics with coupling to an external bath. J Chem Phys 81:3684-3690 
46. Ryckaert JP, Ciccotti G, Berendsen HJ (1977) Numerical integration of the cartesian equations of motion of a system with constraints: molecular dynamics of $n$-alkanes. $\mathbf{J}$ Comput Chem 23:327-341

47. Kollman PA, Massova I, Reyes C, Kuhn B, Huo S, Chong L, Lee M, Lee T, Duan Y, Wang W, Donini O, Srivasan J, Case DA, Cheatam III TE (2000) Calculating structures and free energies of complex molecules: combining molecular mechanics and continuum models. Acc Chem Res 33:889-897

48. Tsui V, Case DA (2001) Theory and applications of the generalized born solvation model in macromolecular simulations. Nucleic Acids Sci 56:275-291

49. Weiser J, Shemkin PS, Still WC (1999) Approximate atomic surfaces from linear combinations of pairwise overlaps. J Comput Chem 20:217-230

50. Pearlman DA (2005) Evaluating the molecular mechanics poisson-boltzmann surface area free energy method using a congeneric series of ligands to p38 MAP kinase J Med Chem 48:7796-7807

51. Zang X, Li X, Wang R (2009) Interpretation of the binding affinities of PTP1B inhibitors with the MM-GB/SA method and the X-Score scoring function. J Chem Inf Model 49:1033-1048

52. Laskowski RA, MacArthur MW, Moss DS, Thornton JM (1993) PROCHECK: a program to check the stereochemical quality of protein structures. J Appl Cryst 26:283291

53. Eisenberg D, Luthy R, Bowie JU (1997) VERIFY3D: assessment of protein models with three-dimensional profiles. Methods Enzymol 277:396-404

54. Wiederstein M, Sippl MJ (2007) ProsaWeb: interactive web service for the recognition of errors in three-dimensional structures of proteins. Nucleic Acids Res 35:407-410

55. Colovos C, Yeates TO (1993) Verification of protein structures: patterns of nonbonded atomic interactions. Protein Sci 2:1511-1519

56. Hirsch AKH, Lauw S, Gersbach P, Schweizer WB, Rohdich F, Eisenreich W, Bacher A, Diederich F (2007) Nonphosphate inhibitors of IspE protein, a kinase in the nonmevalonate pathway for isoprenoid biosynthesis and a potential target for antimalarial therapy. Chemmedchem 2:806-810

57. Gohlke H, Kiel C, Case DA (2003) Insights into protein-protein binding by binding free energy calculations and free energy decomposition for Ras-Raf and Ras-RaIGDS complexes. Mol Biol 330:891-913 
58. Obiol-Pardo C, Rubio-Martinez J (2008) Homology modeling of human transketolase: description of critical sites useful for drug design and study of the cofactor binding mode. J Mol Graph Model 27:723-734

59. Suhre K, Sanejouand YH (2004) ElNemo: a normal mode web-server for protein movement analysis and the generation of templates for molecular replacement. Nucleic Acids Res 32:W610-W614

60. Yaffe E, Fishelovitch D, Wolfson HJ, Halperin D, Nussinov (2008) MolAxis: efficient and accurate identification of channels in macromolecules. Prot Struct Funct Bioinform 73:72-86

61. Humprey W, Dalke A, Schulten K (1996) VMD visual molecular dynamics. J Mol Graph $14: 33-38$ 
Table 1 MMGBSA 'per residue' decomposition showing the main residues that contribute to the CDP-methylerythritol binding. Asterisk denotes for a residue of the second monomer. CDP-ME denotes the CDP-methylerythritol molecule

\begin{tabular}{|c|c|}
\hline Residue & $\boldsymbol{A G B}_{\text {Tот }}(\mathbf{k c a l} / \mathbf{m o l})$ \\
\hline P13 & -2.00 \\
\hline A14 & -2.34 \\
\hline A15 & -1.57 \\
\hline G16 & -2.05 \\
\hline T86 & -1.07 \\
\hline A108 & -1.82 \\
\hline R110 & -8.30 \\
\hline D193 & 4.39 \\
\hline K219 & -2.46 \\
\hline Mg & -38.81 \\
\hline CDP-ME & -63.38 \\
\hline T141* & -1.55 \\
\hline
\end{tabular}


Table 2 MolAxis [60] results for the homodimer of M. tuberculosis CMS, showing the ranked channels found and the bottleneck residues involved in these channels. Residues of the second monomer are marked with asterisks. Underlined residues are conserved with respect to the E. coli variant

\begin{tabular}{|c|c|}
\hline Ranked Channel & “Bottleneck” Amino Acids \\
\hline 1 & $\mathrm{~L} 220, \underline{\mathrm{L} 222}, \mathrm{P} 211^{*}, \underline{\mathrm{K} 215^{*}}$ \\
\hline 2 & $\mathrm{Q} 164, \underline{\mathrm{K} 143^{*}}, \mathrm{Q} 164^{*}$ \\
\hline 3 & $\underline{\mathrm{T} 141, \mathrm{Q} 164, \mathrm{Q} 164^{*}}$ \\
\hline 4 & $\mathrm{~A} 213, \underline{\mathrm{D} 221}{ }^{*}$ \\
\hline 5 & $\underline{\mathrm{K} 215^{*}, \underline{\mathrm{T}}^{*}}$ \\
\hline 6 & $\underline{\mathrm{T} 217}, \underline{\mathrm{D} 140^{*}}, \underline{\mathrm{T} 141^{*}}$ \\
\hline 7 & $\mathrm{~V} 118, \mathrm{~V} 135, \mathrm{P} 211$ \\
\hline 8 & $\underline{\mathrm{D} 61, \mathrm{~T} 63, \mathrm{D} 64}$ \\
\hline 9 & $\underline{\mathrm{T} 141}, \underline{\mathrm{P} 166} *, \mathrm{~K} 219^{*}$ \\
\hline 10 & $\mathrm{~K} 219, \mathrm{~L} 220, \mathrm{~L} 138^{*}$ \\
\hline
\end{tabular}




\section{Figure captions}

Fig. 1 Sequence alignment between E. coli CMS (up) and M. tuberculosis CMS (down). Secondary structure prediction using STRIDE is also shown: red accounts for alpha helix, gray accounts for beta sheet, blue accounts for 3-10 helix and yellow accounts for $\pi$-helix

Fig. 2 A) Superimposition of six X-Ray structures that can be used as templates; the $E$. coli variant (1INI) is shown in gray, the Thermotoga maritima variant (1VPA) is shown in purple, the Thermus thermophilus variant (2PX7) is shown in cyan, the Campylobacter jejuni variant (1W55) is shown in red (note that this enzyme has a domain which cannot be used), the Listeria monocytogenes variant (3F1C) is shown in dark blue and the Streptococcus pneumoniae variant (2VSH) is shown in green.

B) Superimposition between the E. coli CMS template (gray) and the best model of M. tuberculosis generated with MODELLER (orange). Superimposed CDPmethylerythritol molecules are also shown. Figures created with the Chimera software [38]

Fig. 3 Verify 3D score profiles [53] for the generated homodimer of M. tuberculosis extracted from MODELLER (dashed lines) or refined through molecular dynamics. Scores over 0.2 indicate a high-quality structure. Asterisks denote for the residues of the second monomer

Fig. 4 ProSa energy scores [54] for the experimental E. coli CMS used as template (A), the MODELLER model of M. tuberculosis CMS (B) and the refined model of $M$. tuberculosis CMS (C). Negative scores indicate a high-quality structure

Fig. 5 A) MODELLER structure of $M$. tuberculosis CMS (only shown one monomer for clarity) marking three loops (in green) which structures were refined as suggested by the scoring functions applied. B) Detailed structure of loop 14-26 before (green) and after (blue) molecular dynamics. Figures created with the VMD software [61] 
Fig. 6 Structures of the substrates MEP and CTP showing the calculated affinities in $M$. tuberculosis CMS as reported in reference [24]

Fig. 7 Detailed view of the most important residues implicated in hydrogen bonds to the CDP-methylerythritol binding and of the magnesium coordination. Asterisk denotes for a residue of the second monomer

Fig. 8 Estimated binding free energy components, averaged over 100 snapshots, for the CDP-methylerythritol bound to the $M$. tuberculosis model ( $\mathrm{kcal} \mathrm{mol}^{-1}$ units). $\Delta \mathrm{E}$ ELE and $E_{\mathrm{VDW}}$ account for the electrostatic and van der Waals in vacuo binding enthalpic contribution. $\triangle \mathrm{GB}$ SUR accounts for the non-polar contribution to solvation, $\Delta \mathrm{GB}$ is the polar contribution to solvation. $\Delta \mathrm{GB}$ SOL denotes for the $\Delta \mathrm{GB}$ SUR $+\Delta \mathrm{GB}_{\mathrm{CAL}}$ addition. $\Delta \mathrm{GB}$ ELE accounts for the $\Delta \mathrm{E}_{\mathrm{ELE}}+\Delta \mathrm{GB}$ CAL addition. $\Delta \mathrm{GB}$ тот accounts for total enthalpic contribution, including the solvation, to the binding free energy using the Generalized Born framework. -T $\Delta \mathrm{S}$ TRAS denotes for the translational entropic contribution, $-\mathrm{T} \Delta \mathrm{S}_{\mathrm{ROT}}$ denotes for the rotational entropic contribution, $-\mathrm{T} \Delta \mathrm{S}_{\mathrm{VIB}}$ accounts for the vibrational entropic contribution and $-\mathrm{T} \Delta \mathrm{S}$ тот accounts for the total entropic balance (all entropic components are calculated truncating the receptor to only those atom within a cutoff of $9 \AA$ to the ligand and using 10 snapshots). Final estimation of the theoretical binding free energy at 300 $\mathrm{K}$ is denoted as $\Delta \mathrm{G}_{\text {MMGBSA }}$

Fig. 9 MMGBSA 'per residue' decomposition only showing the residues that contribute most to the dimer stabilization. CDP-ME denotes the CDP-methylerythritol molecule

Fig. 10 Calculated B-factors for the first monomer of M. tuberculosis CMS refined through molecular dynamics

Fig. 11 Most important gating residues involved in channels as defined by MolAxis [60]. Residues of channel 1 are shown in blue, of channel 2 in red, of channel 3 in gray, of channel 4 in yellow, of channel 5 in tan, of channel 6 in white, of channel 7 in pink, of channel 8 in cyan, of channel 9 in purple and of channel 10 in black. CDPmethylerythritol molecules are shown in ball and stick representation. The inset 
gives a detailed view of the best scored channel showing the gating residues, asterisks denote for residues of the second monomer. Figure created with the VMD software [61] 
E. coli CMS

M. tuberculosis CMS

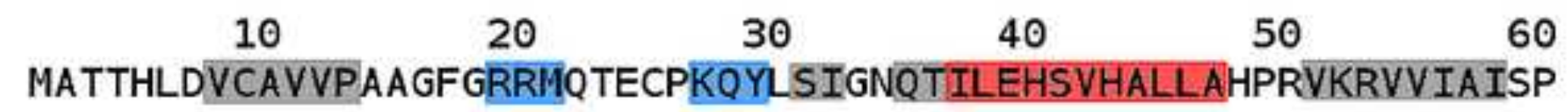

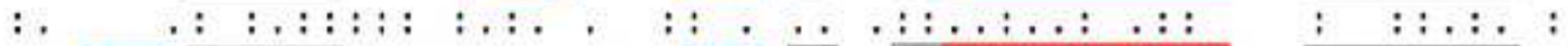
MVREAGEVVAIVPAAGSGERLAVGVPKAFYQLDGQTLIERAVDGLLDSGVVDTVVVAV-P $\begin{array}{lllll}10 & 20 & 30 & 40 & 50\end{array}$ $\begin{array}{lllll}70 & 80 & 90 & 100 & 110\end{array}$ GDSRFAQLPLANHPQITVVDGGDERADSV- - -LAGLKAAGDAQWVLVHDAARPCLHQDDL ADRTDEARQILGH-RAMIVAGGSNRTDTVNLALTVLSGTAEPEFVLVHDAARALTPPALV $\begin{array}{llllll}60 & 70 & 80 & 90 & 100 & 110\end{array}$

$\begin{array}{llllll}120 & 130 & 140 & 150 & 160 & 170\end{array}$

ARLLALSETSRTGGILAAPVRDTMKRAEPGKNAIAHTVDRNGLWHALTPQFFPRELLHDC ARVVEALRDGYAAVVPVLPLSDTIK-AVDANGVVLGTPERAGLRAVQTPQGFTTDLLLRS 120 130 140 150 160 170

180 200 210 220 230 LTR-ALN-EGATITDEASALEYCGFHPQLVEGRADNIKVTRPEDLALAEFYLTRTIHQEN

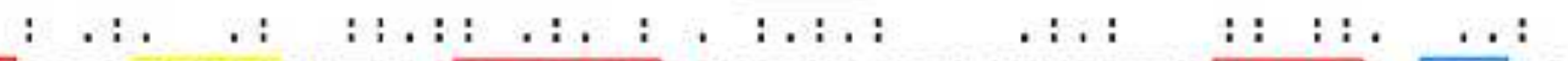
YQRGSLDLPAAEYTDDASLVEHIGGQVQVVDGDPLAFKITTKLDLLLAQ - AIVRG - . . . 180 190 200 210 220 230 
Click here to download high resolution image

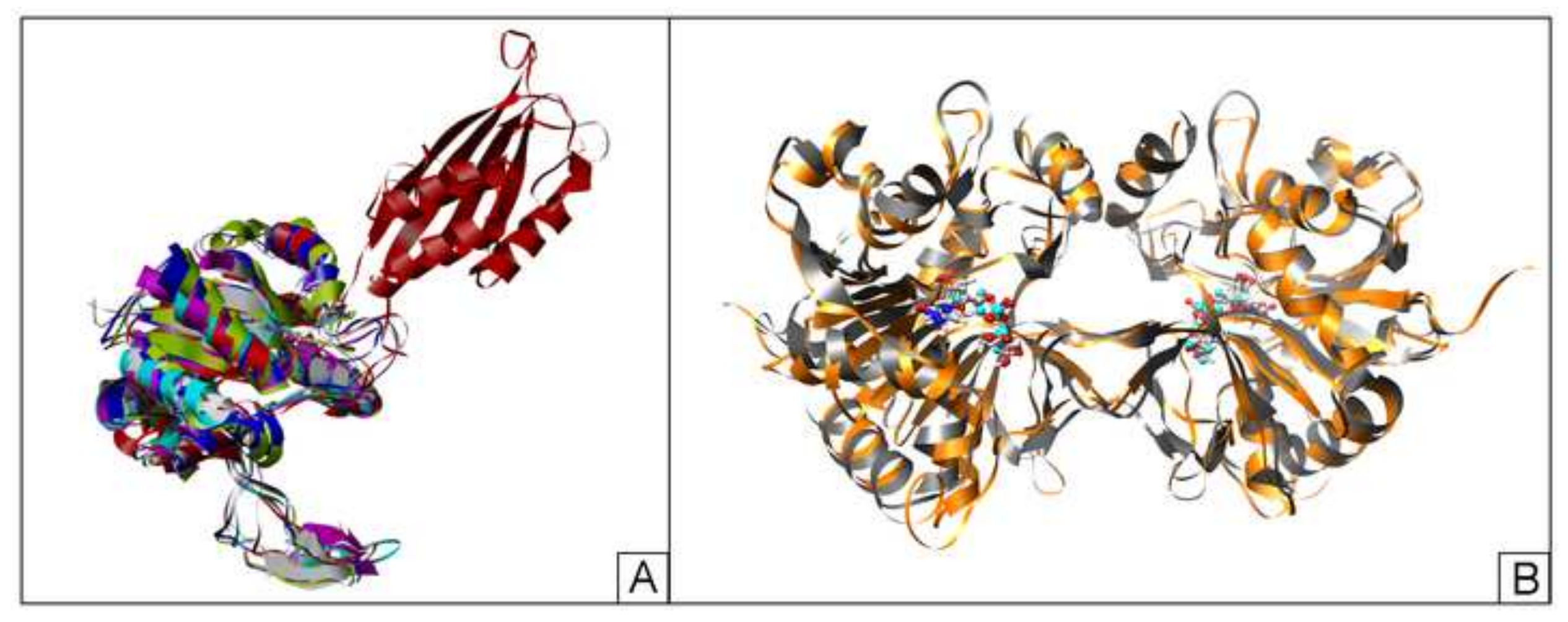

Click here to download high resolution image 


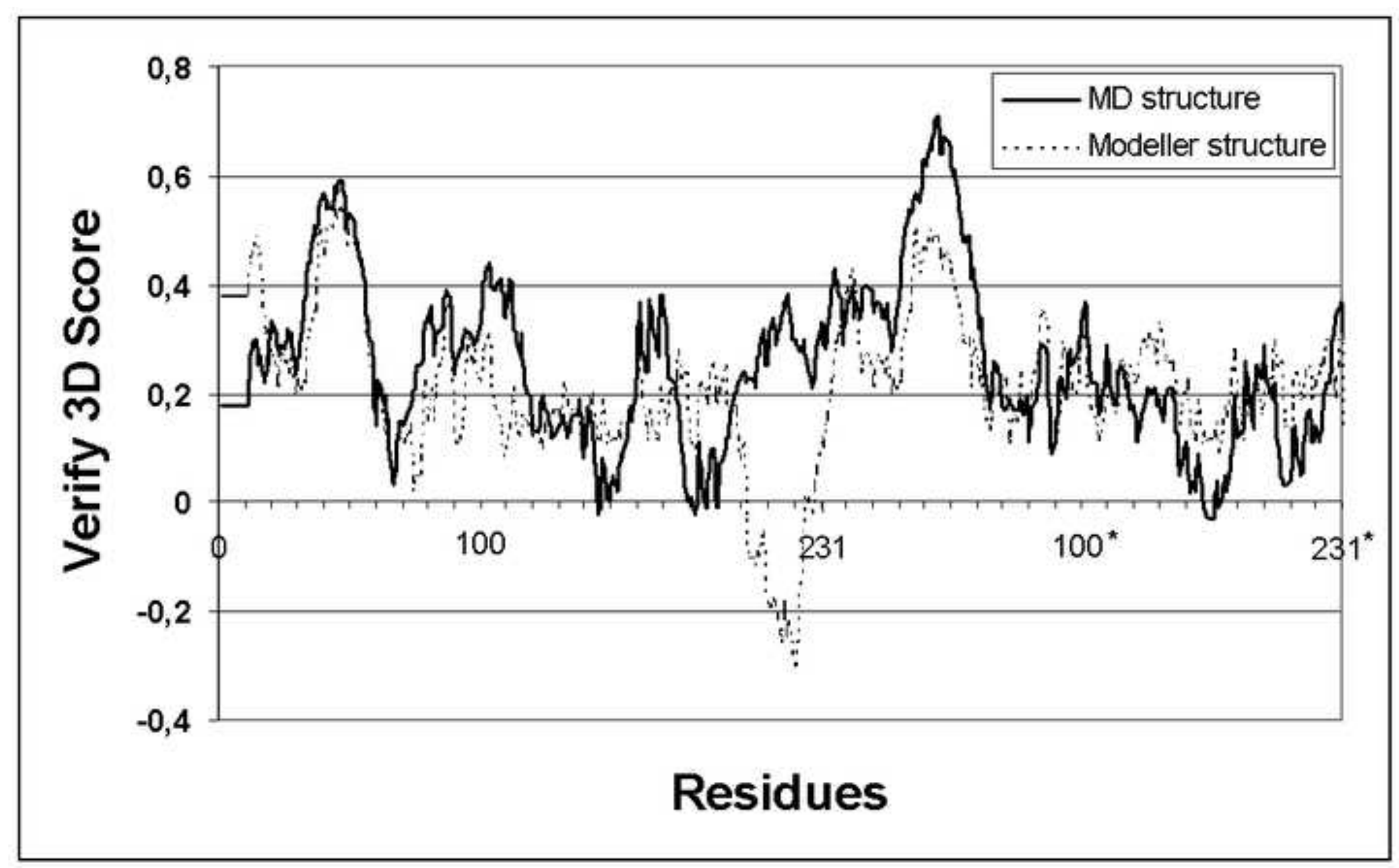

Residues 
Figure 4
Click here to download high resolution image
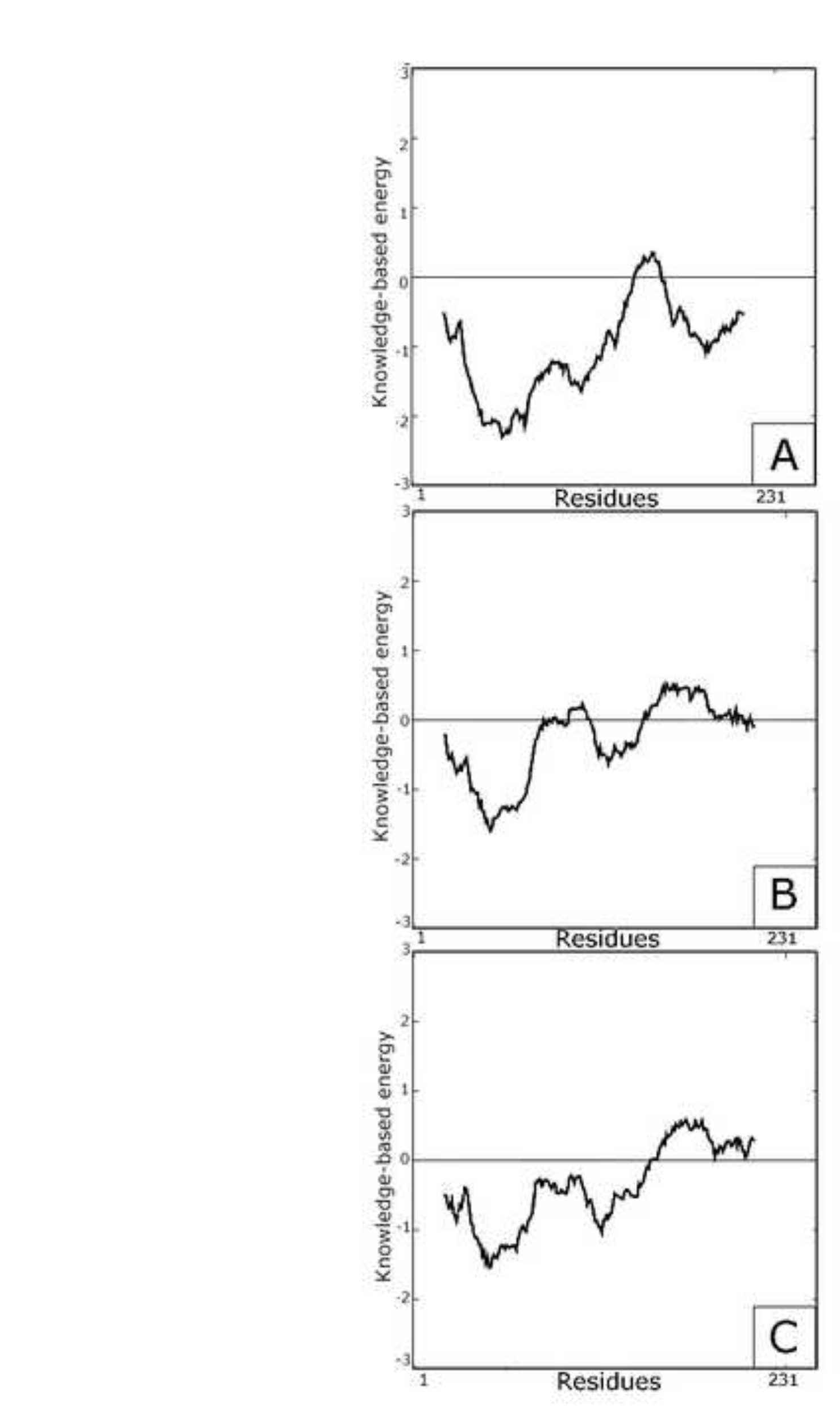


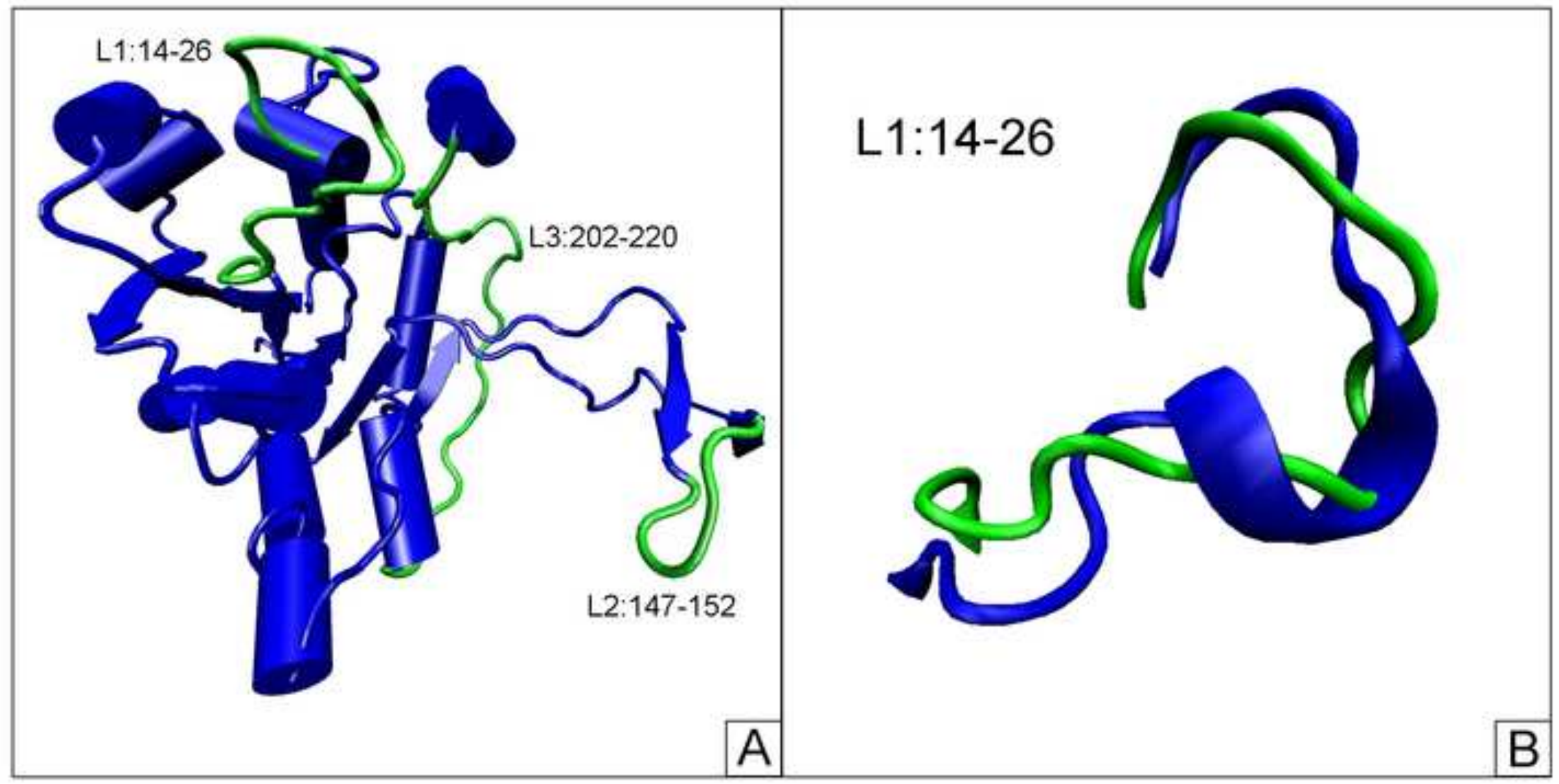



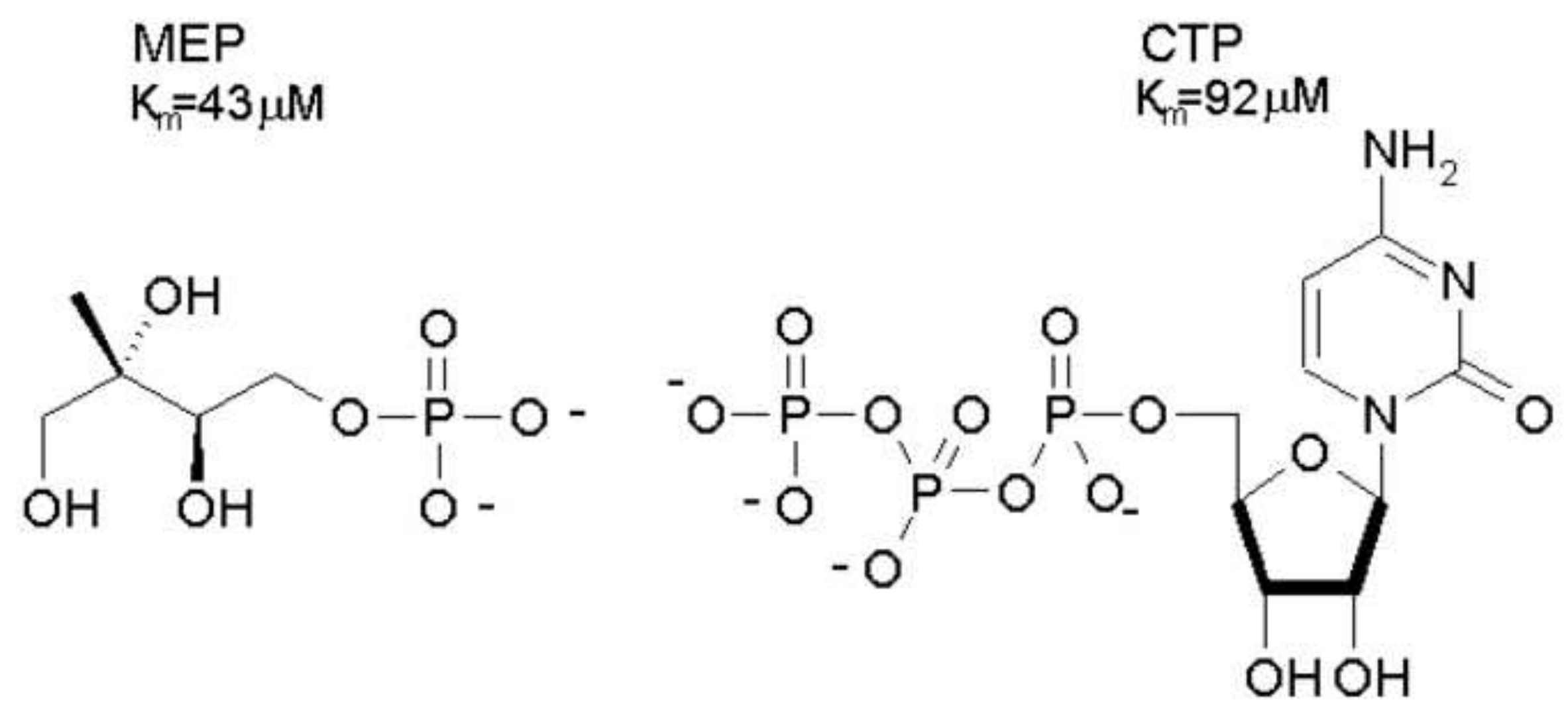


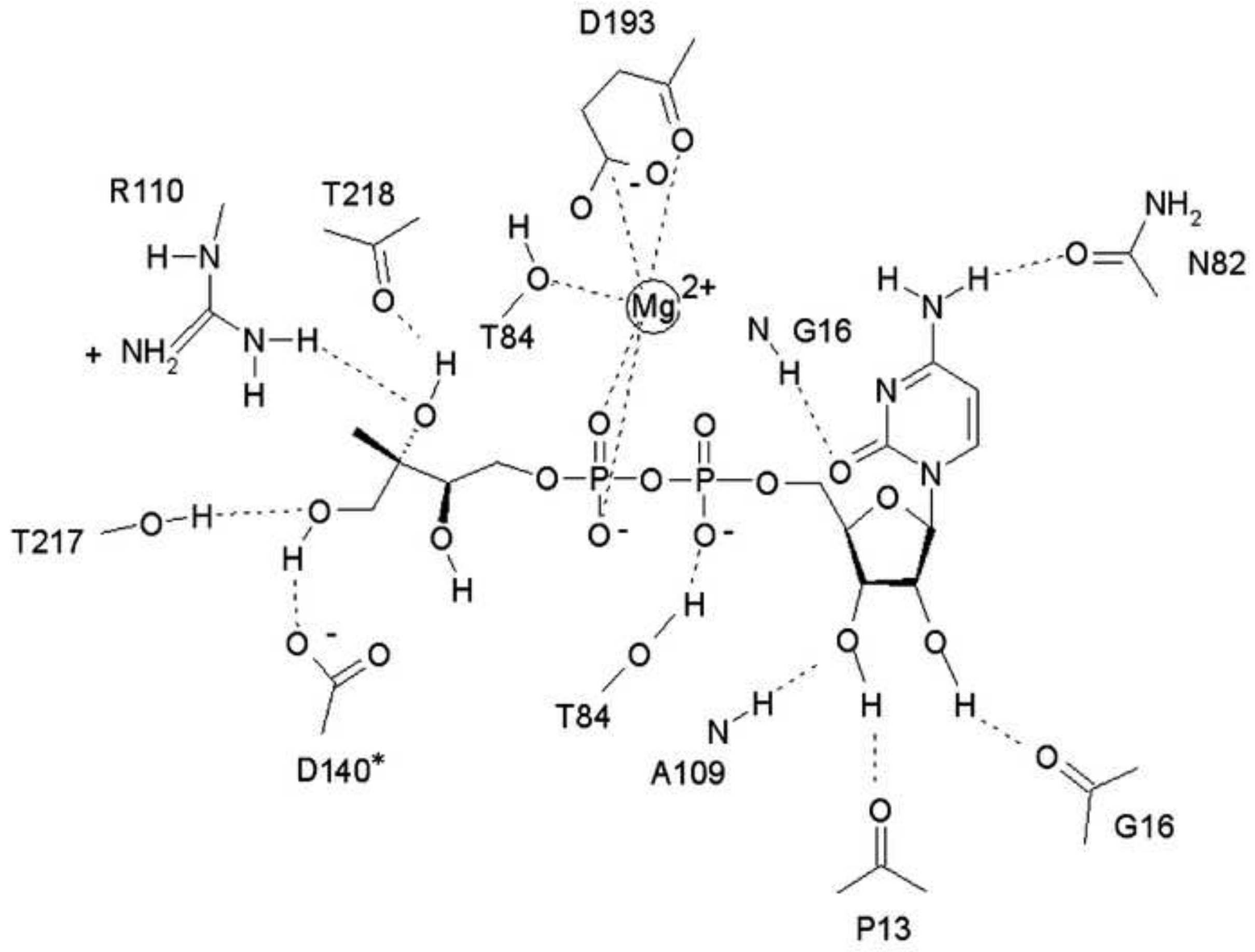




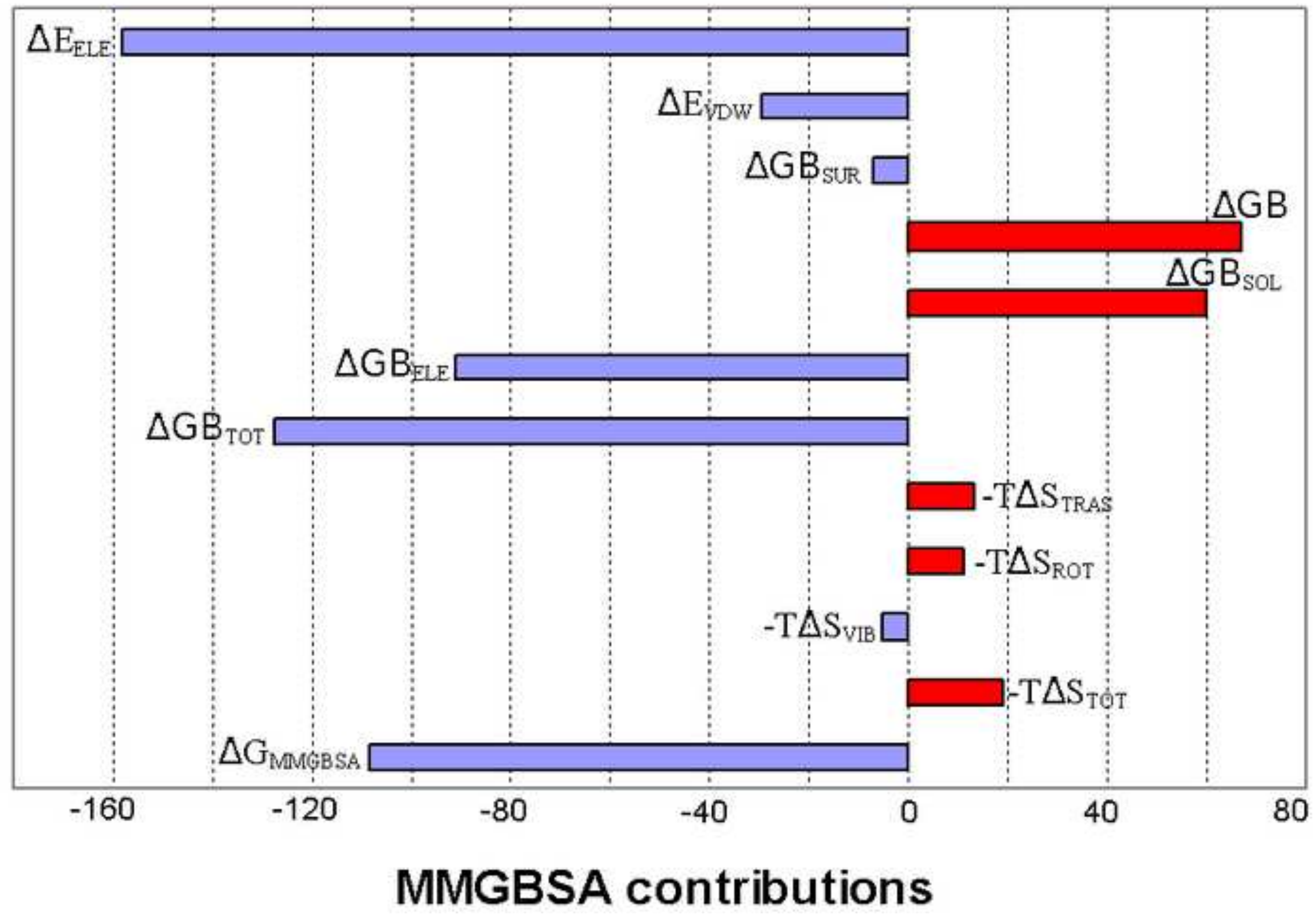




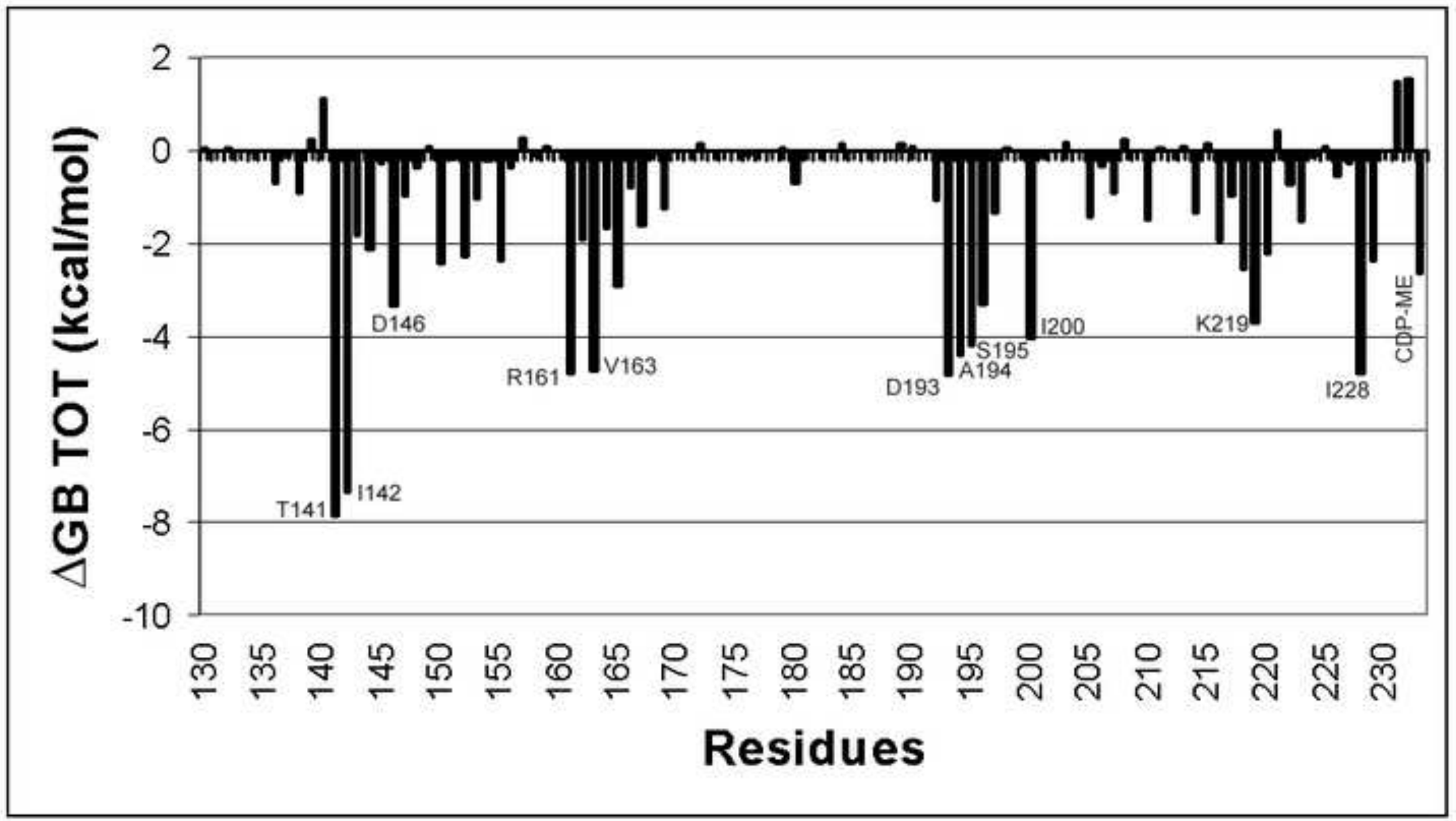




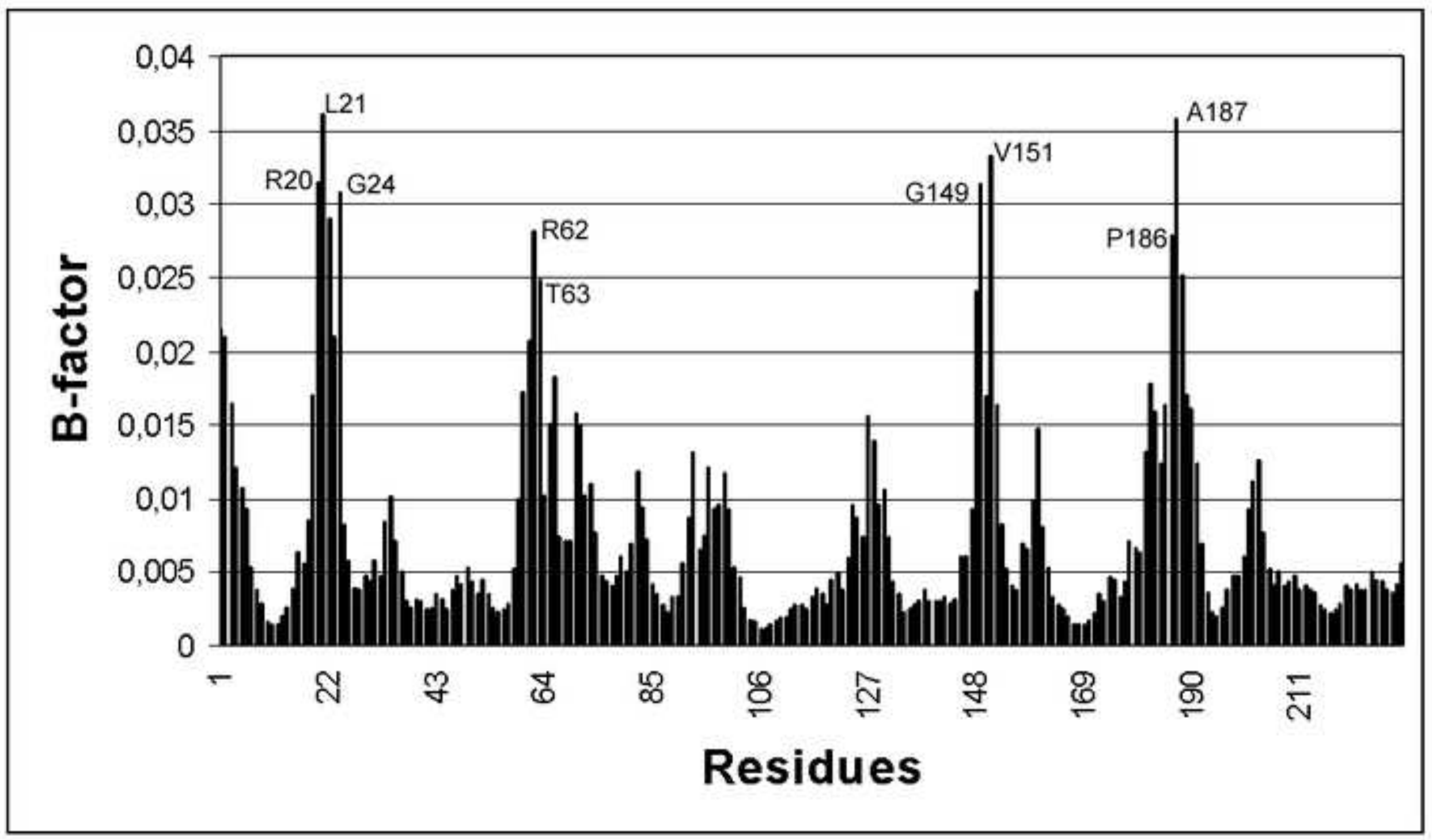


Click here to download high resolution image

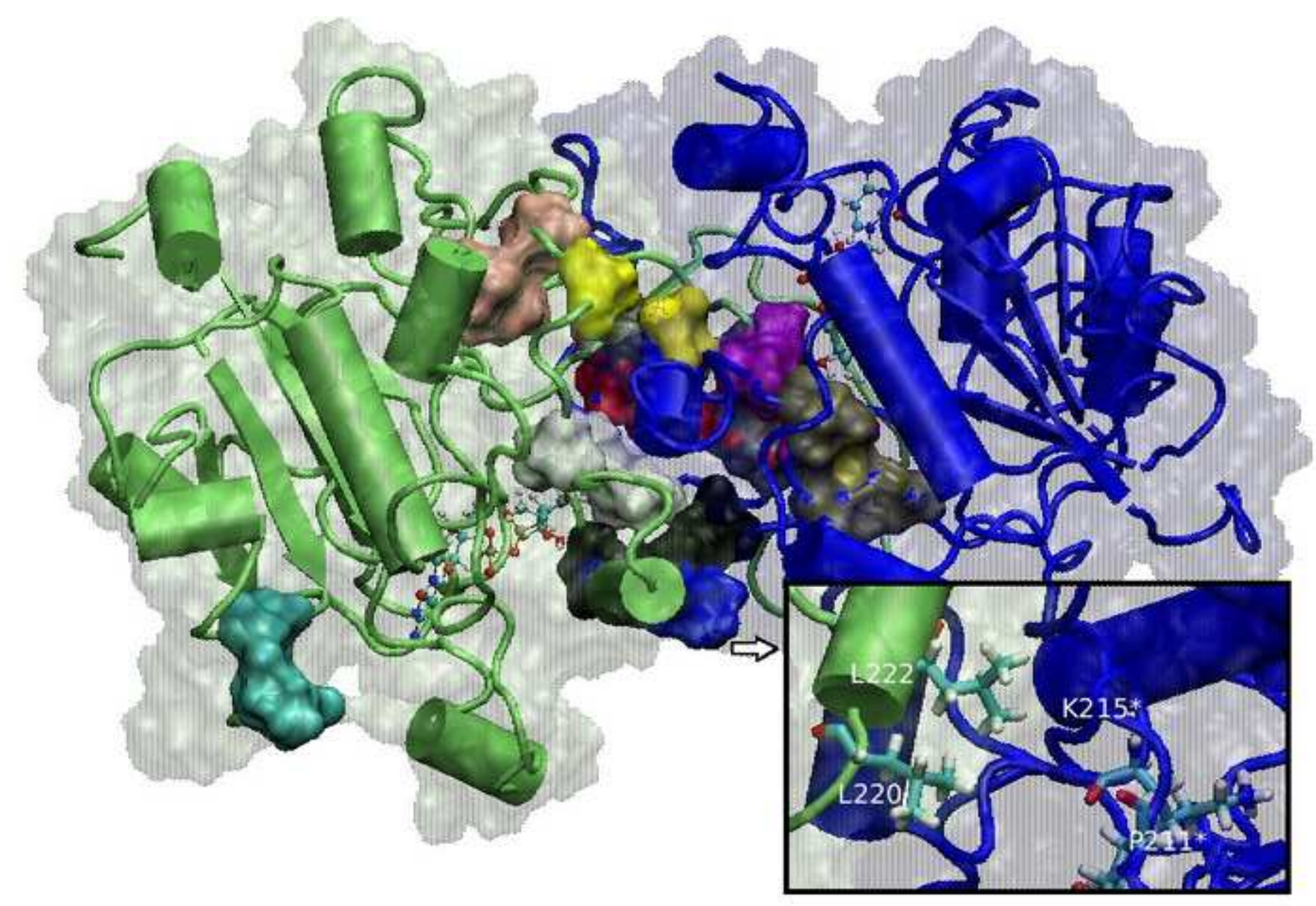

